\title{
A constrained transport divergence-free finite element method for Incompressible MHD equations
}

\author{
Lingxiao Li ${ }^{*} \quad$ Donghang Zhang ${ }^{\dagger} \quad$ Weiying Zheng ${ }^{\ddagger}$
}

\begin{abstract}
In this paper we study finite element method for three-dimensional incompressible resistive magnetohydrodynamic equations, in which the velocity, the current density, and the magnetic induction are divergence-free. It is desirable that the discrete solutions should also satisfy divergence-free conditions exactly especially for the momentum equations. Inspired by constrained transport method, we devise a new stable mixed finite element method that can achieve the goal. We also prove the well-posedness of the discrete solutions. To solve the resulting linear algebraic equations, we propose a GMRES solver with an augmented Lagrangian block preconditioner. By numerical experiments, we verify the theoretical results and demonstrate the quasi-optimality of the discrete solver with respect to the number of degrees of freedom.
\end{abstract}

Key words. Magnetohydrodynamic equations, constrained transport, magnetic vector potential, divergence-free finite element method, block preconditioner.

\section{Introduction}

The magnetohydrodynamic (MHD) equations use fluid theory to describe the interaction of charged particle under the influence of magnetic field. They have broad scientific and engineering applications, such as magnetic fusion [31, astrophysics [23, 46] and liquid metals [17, 12]. The MHD model is a typical multi-scale and multi-physics system. The flow of conducting fluid modifies electromagnetic fields, and conversely the electromagnetic fields modify the momentum of fluid through Lorentz force. The strong coupling between fluid and electromagnetic fields makes the design of effective numerical methods and scalable iterative solvers very difficult (see e.g. 37, 41, 32, 20, 42, 9] and references therein).

In this paper, we study the stationary incompressible resistive MHD equations in a bounded, simplyconnected, and Lipschitz polyhedral domain $\Omega \subset \mathbb{R}^{3}$. They comprise incompressible Navier-Stokes equations and stationary Maxwell's equations

$$
\begin{aligned}
\rho \boldsymbol{u} \cdot \nabla \boldsymbol{u}+\nabla p-\nu \Delta \boldsymbol{u}-\boldsymbol{J} \times \boldsymbol{B}=\boldsymbol{f} & \text { in } \Omega, \\
\operatorname{curl} \boldsymbol{H}=\boldsymbol{J}, \quad \operatorname{curl} \boldsymbol{E}=\mathbf{0} & \text { in } \Omega, \\
\operatorname{div} \boldsymbol{u}=0, \quad \operatorname{div} \boldsymbol{B}=0, \quad \operatorname{div} \boldsymbol{J}=0 & \text { in } \Omega,
\end{aligned}
$$

where $\rho$ is the fluid density, $\boldsymbol{u}$ the fluid velocity, $p$ the hydrodynamic pressure, $\boldsymbol{E}$ the electric feild, $\boldsymbol{H}$ the magnetic field, $\boldsymbol{B}$ the magnetic induction, $\boldsymbol{J}$ the electric current density, and $\boldsymbol{f} \in \boldsymbol{L}^{2}(\Omega)$ external force. The equations in (1) are complemented with the following constitutive equation and generalized Ohm's law

$$
\boldsymbol{B}=\mu \boldsymbol{H}, \quad \boldsymbol{J}=\sigma(\boldsymbol{E}+\boldsymbol{u} \times \boldsymbol{B}) .
$$

The physical parameters are, respectively, dynamic viscosity $\nu$, magnetic permeability $\mu$, and electric conductivity $\sigma$. For the well-posedness of (1) and (2), we assume homogeneous Dirichlet boundary conditions

$$
\boldsymbol{u}=\mathbf{0}, \quad \boldsymbol{H} \times \boldsymbol{n}=\mathbf{0} \quad \text { on } \Gamma:=\partial \Omega .
$$

\footnotetext{
*Institute of Applied Physics and Computational Mathematics, Beijing, 100094, China.

${ }^{\dagger}$ LSEC, NCMIS, Institute of Computational Mathematics and Scientific/Engineering Computing, Academy of Mathematics and Systems Science, Chinese Academy of Sciences, Beijing 100190, China; School of Mathematical Science, University of Chinese Academy of Sciences, Beijing 100049, China.

${ }^{\ddagger}$ LSEC, NCMIS, Institute of Computational Mathematics and Scientific/Engineering Computing, Academy of Mathematics and Systems Science, Chinese Academy of Sciences, Beijing 100190, China; School of Mathematical Science, University of Chinese Academy of Sciences, Beijing 100049, China.
} 
For computational MHD, it is desirable to study discrete methods which respect the divergence-free constraint for magnetic induction, namely $\operatorname{div} \boldsymbol{B}_{h}=0$ 6, 53. There are already many important works on this topic in the literature. For early works one can refer to the review paper [53] and the references therein, where seven schemes are reviewed in detail, such as the 8-wave formulation, the projection scheme, constrained transport (CT) methods and so on. One important observation of the work is the close relation between vector potential method (cf. e.g. [47]) and CT methods (see [14]). In [44], Rossmanith proposed an unstaggered and high-resolution CT method for MHD flows. The readers are also referred to more recent papers [16, 7, 29, 24] which deal with $\operatorname{div} \boldsymbol{B}_{h}=0$ exactly. The second constraint to be satisfied is $\operatorname{div} \boldsymbol{u}_{h}=0$, that is, mass conservation of fluid. Under certain extreme situations, nonphysical phenomena may appear if discrete solutions are not mass-conservative (see 30 for comprehensive discussions).

The third constraint is $\operatorname{div} \boldsymbol{J}_{h}=0$, that is, charge conservation. Assuming small magnetic Reynolds number, the full MHD equations can reduce to a inductionless MHD equations, see [37, 38, 54, 33. In this special case, when the applied magnetic filed is constant, the discrete Lorentz force in the momentum equations, which is a volume force, can only precisely conserve the total momentum when the current density is divergence-free. The authors in 37, 38, 54, suggested that only the numerical schemes, which can conserve the total momentum in the discrete level, can obtain accurate result for MHD flows, especially at large Hartmann numbers. Based on the above considerations, we are motivated to develop a stable numerical scheme which satisfies the three divergence-free conditions simultaneously in the momentum equation, namely,

$$
\operatorname{div} \boldsymbol{u}_{h}=0, \quad \operatorname{div} \boldsymbol{J}_{h}=0, \quad \operatorname{div} \boldsymbol{B}_{h}=0 .
$$

holds in the discrete scheme of momentum equation (1a).

In this paper, we propose a monolithic CT finite element method for (1) such that the discrete solutions in the momentum equations (1a) satisfy (4) exactly. Compared with traditional CT methods, the new method treats magnetic field $\boldsymbol{H}$ and magnetic vector potential $\boldsymbol{A}$ as individual variables by means of edge finite element discretization [36, which doesn't need a staggered grid. The discrete current density $\boldsymbol{J}_{h}:=\operatorname{curl} \boldsymbol{H}_{h}$ and the discrete magnetic induction $\boldsymbol{B}_{h}:=\operatorname{curl} \boldsymbol{A}_{h}$ are divergence-free naturally. As remarked in [6, 14, 37, 38, numerical methods satisfying $\operatorname{div} \boldsymbol{B}_{h}=\operatorname{div} \boldsymbol{J}_{h}=0$ in the momentum equations can reduce nonphysical effects created by the discrete Lorentz force $\boldsymbol{J}_{h} \times \boldsymbol{B}_{h}$ on the fluid movement. To fulfill the first constraint in (4), we adopt precisely the same ideas as in [10, 20, where the velocity $\boldsymbol{u}$ and the pressure $p$ are discretized by $\boldsymbol{H}(\operatorname{div}, \Omega)$-conforming finite elements and fully discontinuous finite elements respectively. The standard mixed finite element methods can ensure $\left\|\operatorname{div} \boldsymbol{u}_{h}\right\|_{L^{2}}=0$ on the discrete level [20].

Now we introduce some but not at all complete references on finite element methods for incompressible MHD equations. More work can be found in the references of the cited papers. In [47, Salah, Soulaimani, and Habashi used $(\boldsymbol{u}, p, \boldsymbol{B}, \boldsymbol{A}, \psi)$ as solution variables where $\psi$ is the scalar electric potential, while adopted Galerkin-least-squares variational formulation and continuous finite element discretization. In [18], Gerbeau developed a stabilized finite element method for the incompressible MHD equations. For stationary full MHD equations, Schneebeli and Schötzau 51 proposed a new mixed finite element method where $\boldsymbol{B}$ is discretized by Nédélec's edge elements. In 2004, Schötzau proved optimal error estimates of the finite element method [52. In 2010, Greif et al extended the work in [52] by discretizing $\boldsymbol{u}$ with $\boldsymbol{H}(\operatorname{div}, \Omega)$ conforming face elements so that $\operatorname{div} \boldsymbol{u}_{h}=0$ holds exactly 20. Our choice in this paper for velocity discretization is exactly the same as 20. In 2008, Prohl proved the convergence and error estimates of finite element method for time-dependent MHD equations where $\boldsymbol{B}$ is discretized with $\boldsymbol{H}(\mathbf{c u r l}, \Omega)$ conforming edge elements [43. In 2017, Hu et al proposed a stable finite element method which discretizes $\boldsymbol{B}$ with face elements and $\boldsymbol{E}$ with edge element so that $\operatorname{div} \boldsymbol{B}_{h}=0$ holds exactly [29]. In 2018, Hiptmair et al proposed a new stable finite element method using $(\boldsymbol{u}, p, \boldsymbol{A})$ so that the discrete velocity and the discrete magnetic induction are both divergence-free exactly. For finite element error estimates, we would also like to mention 27] for Euler semi-implicit scheme and [49 for penalty-based finite element methods. We remark that, for $\boldsymbol{B}$-based formulations like in 22, 51, 52, 43, 27] using edge element or nodal element, the discrete current density $\boldsymbol{J}_{h}:=\operatorname{curl} \boldsymbol{B}_{h}$ in the moment equation is divergence-free exactly. We also refer to [38, 54, 33] for charge-conservative methods for inductionless MHD equations where the applied magnetic induction is known in advance.

The second objective of this paper is to propose a monolithic iterative solver with augmented block preconditioner for the discrete problem linearized by Picard's method. We refer to the monograph of Elman, Silvester, and Wathen [13] for a comprehensive introduction of preconditioners and iterative solvers for solving incompressible Navier-Stokes equations. For MHD equations, there are extensive studies in the literature, such as [41, 9, 32, 40, on block preconditioners based on approximate Schur complements. We refer to [48, 50, 34] for algebraic multigrid methods and to [2] for geometric multigrid method. In [8], 
Badia, Martín, and Planas proposed block recursive LU preconditioners for solving thermally coupled inductionless MHD equations. In the present paper, we follow similar ideas as in 41, 32, to propose an augmented Lagrangian block preconditioner for solving the discrete problem. Since div $\boldsymbol{u}_{h}=0$ in our case, the augmented term $\alpha\left(\operatorname{div} \boldsymbol{u}_{h}, \operatorname{div} \boldsymbol{v}_{h}\right)$ added to the momentum equation does not modify the discrete problem, but enhances the robustness of the block preconditioner. Numerical examles show that the convergence of preconditioned GMRES solver is quasi-uniform to the number of degrees of freedom (DOFs).

The paper is organized as follows: In Section 2, we derive a mixed formulation for the full MHD model by means of constrained transport method. In Section 3, we propose a stable mixed finite element method for the incompressible MHD model such that $\boldsymbol{u}_{h}, \boldsymbol{J}_{h}$, and $\boldsymbol{B}_{h}$ are divergence-free exactly in the momentum equations. A Picard-type linearization for the nonlinear discrete problem is also proposed and the well-posedness of the linearized problem is proven. In Section 4, to solve the linearized discrete problem, we propose an augmented Lagrangian block preconditioner by deriving the approximate Schur complements of the MHD system. In Section 5, we present several numerical examples to verify the theoretical results and to demonstrate the performance of the preconditioned monolithic solver. Finally in Section 6, a conclusion is given and some future researches are pointed out. Throughout the paper, we assume that $\rho, \nu, \mu, \sigma$ are positive constants and denote vector-valued quantities by boldface notations, such as $\boldsymbol{L}^{2}(\Omega):=\left(L^{2}(\Omega)\right)^{3}$.

\section{Constrained transport model of MHD equations}

Let $L^{2}(\Omega)$ be the usual Hilbert space of square integrable functions and $H^{1}(\Omega), \boldsymbol{H}(\operatorname{curl}, \Omega), \boldsymbol{H}(\operatorname{div}, \Omega)$ be its subspaces with square integrable gradients, curls, and divergences, respectively. Let $H_{0}^{1}(\Omega)$, $\boldsymbol{H}_{0}(\mathbf{c u r l}, \Omega), \boldsymbol{H}_{0}(\operatorname{div}, \Omega)$ denote their subspaces with vanishing traces, vanishing tangential traces, and vanishing normal traces on $\Gamma$ respectively. We refer to [19] page 26] for their definitions and inner products. For convenience, we also introduce the curl-free and divergence-free subspaces

$$
\begin{aligned}
& \boldsymbol{H}(\operatorname{curl} 0, \Omega)=\{\boldsymbol{v} \in \boldsymbol{H}(\operatorname{curl}, \Omega): \operatorname{curl} \boldsymbol{v}=\mathbf{0}\} \\
& \boldsymbol{H}(\operatorname{div} 0, \Omega)=\{\boldsymbol{v} \in \boldsymbol{H}(\operatorname{div}, \Omega): \operatorname{div} \boldsymbol{v}=0\} .
\end{aligned}
$$

\subsection{Constrained transport formula}

As remarked in [14, Section III], when $\operatorname{div} \boldsymbol{B}_{h} \neq 0$, the discrete Lorentz force $\boldsymbol{J}_{h} \times \boldsymbol{B}_{h}$ may yield inaccurate results in the momentum equation of Navier-Stokes equations. To improve the trustworthiness of $\boldsymbol{B}_{h}$, one should keep the constraint $\nabla \cdot \boldsymbol{B}_{h}=0$ in the discrete level.

The traditional CT method is used to solve ideal magnetic induction equations

$$
\partial_{t} \boldsymbol{B}+\operatorname{curl} \boldsymbol{E}=0, \quad \boldsymbol{E}=\boldsymbol{B} \times \boldsymbol{u}, \quad \operatorname{div} \boldsymbol{B}=0 .
$$

In [14], $\boldsymbol{B}$ and $\boldsymbol{E}$ are discretized on staggered grids, namely, the discretization of $\boldsymbol{B}$ is face-centered and the discretization of $\boldsymbol{E}$ is edge-centered. Since $\operatorname{div} \boldsymbol{B}=0$, there is a magnetic vector potential $\boldsymbol{A}$ such that $\boldsymbol{B}=\operatorname{curl} \boldsymbol{A}$. The CT method amounts to computing the discrete magnetic potential $\boldsymbol{A}_{h}$ by edge-centered discretization and defining the discrete magnetic induction by $\boldsymbol{B}_{h}=\operatorname{curl} \boldsymbol{A}_{h}$. The evolution equation of $\boldsymbol{A}$ is derived from (5) by using temporal gauge $\boldsymbol{E}=-\partial_{t} \boldsymbol{A}$ (see [14, (4.14)-(4.17)])

$$
\partial_{t} \boldsymbol{A}=\boldsymbol{u} \times \operatorname{curl} \boldsymbol{A} .
$$

Similar ideas can also be found in works related to vector potential methods [24, 45, 44].

For the stationary MHD model (1), one faces a major difficulty when dealing with Lorentz force with only magnetic vector potential (see [14])

$$
\boldsymbol{J} \times \boldsymbol{B}=\operatorname{curl} \boldsymbol{H} \times \boldsymbol{B}=\operatorname{curl}\left(\mu^{-1} \operatorname{curl} \boldsymbol{A}\right) \times \operatorname{curl} \boldsymbol{A},
$$

namely, one has to discretize second derivatives of $\boldsymbol{A}$ in the Lorentz force term. To overcome the difficulty, we propose to compute the Lorentz force by

$$
\boldsymbol{J} \times \boldsymbol{B}=\operatorname{curl} \boldsymbol{H} \times \operatorname{curl} \boldsymbol{A}
$$

and discretize $\boldsymbol{H}, \boldsymbol{A}$ as individual variables. This amounts to applying vector potential methods to both $\boldsymbol{J}$ and $\boldsymbol{B}$ simultaneously. 


\subsection{Constrained transport formula of (1)}

From the vector potential theorem in [1, we easily get the following theorem.

Lemma 1. Suppose $\Omega$ is a simply-connected and Lipschitz domain. For any $\boldsymbol{B} \in \boldsymbol{H}(\operatorname{div} 0, \Omega) \cap$ $\boldsymbol{H}_{0}(\mathbf{c u r l}, \Omega)$, there exists a unique $\boldsymbol{A} \in \boldsymbol{H}(\mathbf{c u r l}, \Omega)$ such that

$$
\begin{array}{rll}
\operatorname{curl} \boldsymbol{A}=\boldsymbol{B}, & \operatorname{div} \boldsymbol{A}=0 & \text { in } \Omega, \\
\operatorname{curl} \boldsymbol{A} \times \boldsymbol{n}=0, & \boldsymbol{A} \cdot \boldsymbol{n}=0 & \text { on } \Gamma .
\end{array}
$$

Because $\boldsymbol{B}=\mu \boldsymbol{H}$ and taking curls of the first equation of (7) one obtains

$$
\operatorname{curl} \mu^{-1} \operatorname{curl} \boldsymbol{A}=\operatorname{curl} \boldsymbol{H}, \quad \operatorname{div} \boldsymbol{A}=0 \quad \text { in } \Omega
$$

which is precisely the classical double curl problem for $\boldsymbol{A}$ and can be efficiently solved using existing techniques. Using $\boldsymbol{J}=\operatorname{curl} \boldsymbol{H}$ and eliminating the electric field $\boldsymbol{E}$ we firstly have

$$
\operatorname{curl}\left(\sigma^{-1} \operatorname{curl} \boldsymbol{H}\right)+\operatorname{curl}(\boldsymbol{B} \times \boldsymbol{u})=\mathbf{0} \text { in } \Omega
$$

where $\boldsymbol{B} \times \boldsymbol{u}$ is called induced electric field due to movement of the fluid. Based on (8), then using $\boldsymbol{J}=\operatorname{curl} \boldsymbol{H}$ and $\boldsymbol{B}=\operatorname{curl} \boldsymbol{A}$ both in the Lorentz force $\boldsymbol{J} \times \boldsymbol{B}$ and induced electric field $\boldsymbol{B} \times \boldsymbol{u}$, an equivalent $\mathrm{CT}$ form of (1) can be given as follows

$$
\begin{aligned}
& \rho \boldsymbol{u} \cdot \nabla \boldsymbol{u}+\nabla p-\nu \Delta \boldsymbol{u}-\operatorname{curl} \boldsymbol{H} \times \operatorname{curl} \boldsymbol{A}=\boldsymbol{f} \quad \text { in } \Omega, \\
& \operatorname{curl}\left(\sigma^{-1} \operatorname{curl} \boldsymbol{H}\right)+\operatorname{curl}(\operatorname{curl} \boldsymbol{A} \times \boldsymbol{u})=\mathbf{0} \text { in } \Omega, \\
& \operatorname{curl} \mu^{-1} \operatorname{curl} \boldsymbol{A}-\operatorname{curl} \boldsymbol{H}=\mathbf{0} \quad \text { in } \Omega \text {, } \\
& \operatorname{div} \boldsymbol{u}=0, \quad \operatorname{div}(\mu \boldsymbol{H})=0, \quad \operatorname{div} \boldsymbol{A}=0 \quad \text { in } \Omega, \\
& \boldsymbol{u}=\mathbf{0}, \quad \boldsymbol{H} \times \boldsymbol{n}=\mathbf{0}, \quad \boldsymbol{A} \cdot \boldsymbol{n}=0, \quad \operatorname{curl} \boldsymbol{A} \times \boldsymbol{n}=\mathbf{0} \quad \text { on } \Gamma .
\end{aligned}
$$

The reason we use $\boldsymbol{A}$ to represent the induced electric field is that we want the whole FEM to be stable. This skill has already appeared in the previous work of [22, [51, 52. Remember that $\rho, \nu, \sigma, \mu$ are all positive constants. Let $U, H, L$ be the characteristic quantities for velocity, magnetic field, and length of the system respectively. Define the dimensionless Reynolds number $R_{e}$, coupling number $\kappa$, and magnetic Reynolds number $R_{m}$ by

$$
R_{e}=U L \rho / \nu, \quad \kappa=\mu H^{2} /\left(\rho U^{2}\right), \quad R_{m}=\mu \sigma U L .
$$

Then the MHD system can be written into a dimensionless form

$$
\begin{aligned}
\boldsymbol{u} \cdot \nabla \boldsymbol{u}+\nabla p-R_{e}^{-1} \Delta \boldsymbol{u}-\kappa \operatorname{curl} \boldsymbol{H} \times \operatorname{curl} \boldsymbol{A}=\boldsymbol{f} & \text { in } \Omega, \\
\kappa R_{m}^{-1} \operatorname{curl} \operatorname{curl} \boldsymbol{H}+\kappa \operatorname{curl}(\operatorname{curl} \boldsymbol{A} \times \boldsymbol{u})+\nabla r=\mathbf{0} & \text { in } \Omega, \\
\operatorname{curl} \operatorname{curl} \boldsymbol{A}-\operatorname{curl} \boldsymbol{H}+\nabla \phi=\mathbf{0} & \text { in } \Omega, \\
\operatorname{div} \boldsymbol{u}=0, \quad \operatorname{div} \boldsymbol{H}=0, \quad \operatorname{div} \boldsymbol{A}=0 & \text { in } \Omega, \\
\boldsymbol{H} \times \boldsymbol{n}=\mathbf{0}, \quad \boldsymbol{A} \cdot \boldsymbol{n}=0, \quad \operatorname{curl} \boldsymbol{A} \times \boldsymbol{n}=\mathbf{0} & \text { on } \Gamma, \\
\boldsymbol{u}=\mathbf{0}, \quad r=0, \quad \nabla \phi \cdot \boldsymbol{n}=0 & \text { on } \Gamma .
\end{aligned}
$$

In $(10), r$ and $\phi$ are, respectively, Lagrange multipliers for $\boldsymbol{H}$ and $\boldsymbol{A}$. Taking divergences of $10 \mathrm{~b}$ and (10c) and using (10f), we get

$$
\Delta r=0, \Delta \phi=0 \quad \text { in } \Omega, \quad r=0, \quad \nabla \phi \cdot \boldsymbol{n}=0 \quad \text { on } \Gamma .
$$

This implies $\nabla r=\nabla \phi=0$. So 10 is actually equivalent to (9). Although the overall structure of the new formulation is similar to the ones in [22, 52, 20, here we introduce an extra magnetic vector potential $\boldsymbol{A}$ inspired by original CT methods and therefore ensure the divergence-free conditions for $\boldsymbol{B}_{h}$ in the Lorentz force.

In our previous work we have used vector potential with edge element method in $3 \mathrm{D}$ to represent $\boldsymbol{B}$ [24] but the discrete current density of the Lorentz force there is only weakly divergence-free. In [20], 
$\boldsymbol{u}_{h}$ and $\boldsymbol{J}_{h}$ are divergence-free in the momentum equation. In the present work we incorporate the two advantages of the formulation in [22, 20, and traditional vector potential methods to realize the two divergence-free conditions in the Lorentz force at the same time. However compared with the work in [22, 20, two extra variables $\boldsymbol{A}$ and $\phi$ are incurred. One can see that standard mixed finite element methods will give a triple saddle-point problem which is more difficult to solve. Thus another object of this paper is to develop preconditioned iterative methods to reduce the overall cost as best we can.

\subsection{A weak formulation}

For convenience, we introduce some notations for function spaces

$$
\begin{array}{ccc}
\boldsymbol{V}:=\boldsymbol{H}_{0}^{1}(\Omega), & \boldsymbol{W}:=\boldsymbol{H}_{0}(\operatorname{curl}, \Omega), & \boldsymbol{D}:=\boldsymbol{H}(\operatorname{curl}, \Omega), \\
Q:=L^{2}(\Omega) / \mathbb{R}, & S:=H_{0}^{1}(\Omega), & Y:=H^{1}(\Omega) / \mathbb{R} .
\end{array}
$$

The divergence-free subspaces of $\boldsymbol{V}, \boldsymbol{W}$, and $\boldsymbol{D}$ are defined by

$$
\boldsymbol{U}(\operatorname{div} 0):=\boldsymbol{U} \cap \boldsymbol{H}(\operatorname{div} 0, \Omega) \text { for } \boldsymbol{U}=\boldsymbol{V}, \boldsymbol{W}, \boldsymbol{D} .
$$

Multiplying both sides of $10 \mathrm{a}$ with $\boldsymbol{v} \in \boldsymbol{V}$ and integrating by parts, we get

$$
\mathscr{A}(\boldsymbol{u}, \boldsymbol{v})+\mathscr{O}(\boldsymbol{u} ; \boldsymbol{u}, \boldsymbol{v})-\mathscr{L}(\boldsymbol{A} ; \boldsymbol{v}, \boldsymbol{H})-(p, \operatorname{div} \boldsymbol{v})=(\boldsymbol{f}, \boldsymbol{v}),
$$

where the bilinear form $\mathscr{A}$ and the trilinear forms $\mathscr{O}, \mathscr{L}$ are defined respectively by

$$
\begin{aligned}
& \mathscr{A}(\boldsymbol{w}, \boldsymbol{v}):=R_{e}^{-1}(\nabla \boldsymbol{w}, \nabla \boldsymbol{v}), \quad \mathscr{O}(\boldsymbol{w} ; \boldsymbol{u}, \boldsymbol{v}):=(\boldsymbol{w} \cdot \nabla \boldsymbol{u}, \boldsymbol{v}), \\
& \mathscr{L}(\boldsymbol{A} ; \boldsymbol{v}, \boldsymbol{H}):=\kappa(\operatorname{curl} \boldsymbol{A} \times \boldsymbol{v}, \operatorname{curl} \boldsymbol{H}) .
\end{aligned}
$$

Multiply both sides of $110 \mathrm{~b}$ with $\boldsymbol{w} \in \boldsymbol{W}$ and both sides of $(10 \mathrm{c})$ with $\boldsymbol{d} \in \boldsymbol{D}$. Using integration by parts, we get

$$
\begin{aligned}
& \mathscr{C}_{1}(\boldsymbol{H}, \boldsymbol{w})+\mathscr{L}(\boldsymbol{A} ; \boldsymbol{u}, \boldsymbol{w})+(\nabla r, \boldsymbol{w})=0, \\
& \mathscr{C}(\boldsymbol{A}, \boldsymbol{d})-(\boldsymbol{H}, \operatorname{curl} \boldsymbol{d})+(\nabla \phi, \boldsymbol{d})=0,
\end{aligned}
$$

where the bilinear forms are defined by

$$
\mathscr{C}_{1}(\boldsymbol{v}, \boldsymbol{w}):=\kappa R_{m}^{-1}(\operatorname{curl} \boldsymbol{v}, \operatorname{curl} \boldsymbol{w}), \quad \mathscr{C}(\boldsymbol{v}, \boldsymbol{w}):=(\operatorname{curl} \boldsymbol{v}, \operatorname{curl} \boldsymbol{w}) .
$$

Combining (11)-13), we obtain a weak formulation of 10 :

Find $(\boldsymbol{u}, \boldsymbol{H}, \boldsymbol{A}) \in \boldsymbol{V} \times \boldsymbol{W} \times \boldsymbol{D}$ and $(p, r, \phi) \in Q \times S \times Y$ such that

$$
\begin{aligned}
& \mathscr{A}(\boldsymbol{u}, \boldsymbol{v})+\mathscr{O}(\boldsymbol{u} ; \boldsymbol{u}, \boldsymbol{v})-\mathscr{L}(\boldsymbol{A} ; \boldsymbol{v}, \boldsymbol{H})-(p, \operatorname{div} \boldsymbol{v})=(\boldsymbol{f}, \boldsymbol{v}), \\
& \mathscr{C}_{1}(\boldsymbol{H}, \boldsymbol{w})+\mathscr{L}(\boldsymbol{A} ; \boldsymbol{u}, \boldsymbol{w})+(\nabla r, \boldsymbol{w})=0, \\
& \mathscr{C}(\boldsymbol{A}, \boldsymbol{d})-(\boldsymbol{H}, \operatorname{curl} \boldsymbol{d})+(\nabla \phi, \boldsymbol{d})=0, \\
& (\operatorname{div} \boldsymbol{u}, q)=0, \quad(\boldsymbol{H}, \nabla s)=0, \quad(\boldsymbol{A}, \nabla \varphi)=0,
\end{aligned}
$$

for all $(\boldsymbol{v}, \boldsymbol{w}, \boldsymbol{d}) \in \boldsymbol{V} \times \boldsymbol{W} \times \boldsymbol{D}$ and $(q, s, \varphi) \in Q \times S \times Y$.

Theorem 2. The solutions of (14) satisfy the stability estimate

$$
\|\boldsymbol{u}\|_{\boldsymbol{H}^{1}(\Omega)}+\|\boldsymbol{H}\|_{\boldsymbol{H}(\operatorname{curl}, \Omega)}+\|\boldsymbol{A}\|_{\boldsymbol{H}(\operatorname{curl}, \Omega)} \leq C\|\boldsymbol{f}\|_{\boldsymbol{L}^{2}(\Omega)},
$$

where the constant $C$ depends only on $\kappa, R_{e}, R_{m}$, and the domain $\Omega$.

Proof. Using $(14 \mathrm{~d})$, it is easy to see $\boldsymbol{u} \in \boldsymbol{V}(\operatorname{div} 0), \boldsymbol{H} \in \boldsymbol{W}(\operatorname{div} 0)$, and $\boldsymbol{A} \in \boldsymbol{D}(\operatorname{div} 0) \cap \boldsymbol{H}_{0}(\operatorname{div}, \Omega)$. Then (14) is reduced to

$$
\begin{array}{ll}
\mathscr{A}(\boldsymbol{u}, \boldsymbol{v})+\mathscr{O}(\boldsymbol{u} ; \boldsymbol{u}, \boldsymbol{v})-\mathscr{L}(\boldsymbol{A} ; \boldsymbol{v}, \boldsymbol{H})=(\boldsymbol{f}, \boldsymbol{v}) \quad \forall \boldsymbol{v} \in \boldsymbol{V}(\operatorname{div} 0) \\
\mathscr{C}_{1}(\boldsymbol{H}, \boldsymbol{w})+\mathscr{L}(\boldsymbol{A} ; \boldsymbol{u}, \boldsymbol{w})=0 & \forall \boldsymbol{w} \in \boldsymbol{W}(\operatorname{div} 0), \\
\mathscr{C}(\boldsymbol{A}, \boldsymbol{d})-(\boldsymbol{H}, \boldsymbol{c u r l} \boldsymbol{d})=0 & \forall \boldsymbol{d} \in \boldsymbol{D}(\operatorname{div} 0) .
\end{array}
$$


Taking $(\boldsymbol{v}, \boldsymbol{w}, \boldsymbol{d})=(\boldsymbol{u}, \boldsymbol{H}, \boldsymbol{A})$ and using $\operatorname{div} \boldsymbol{u}=0$, we find that

$$
\begin{aligned}
& \frac{1}{R_{e}}|\boldsymbol{u}|_{\boldsymbol{H}^{1}(\Omega)}^{2}-\mathscr{L}(\boldsymbol{A} ; \boldsymbol{u}, \boldsymbol{H})=(\boldsymbol{f}, \boldsymbol{u}), \quad \frac{\kappa}{R_{m}}\|\operatorname{curl} \boldsymbol{H}\|_{\boldsymbol{L}^{2}(\Omega)}^{2}+\mathscr{L}(\boldsymbol{A} ; \boldsymbol{u}, \boldsymbol{H})=0, \\
& \|\operatorname{curl} \boldsymbol{A}\|_{\boldsymbol{L}^{2}(\Omega)}^{2}=(\boldsymbol{H}, \operatorname{curl} \boldsymbol{A}) .
\end{aligned}
$$

Adding up the first and second equations yields

$$
\frac{1}{R_{e}}|\boldsymbol{u}|_{\boldsymbol{H}^{1}(\Omega)}^{2}+\frac{\kappa}{R_{m}}\|\operatorname{curl} \boldsymbol{H}\|_{\boldsymbol{L}^{2}(\Omega)}^{2}=(\boldsymbol{f}, \boldsymbol{u}) \leq C\|\boldsymbol{f}\|_{\boldsymbol{L}^{2}(\Omega)}^{2}+\frac{1}{2 R_{e}}|\boldsymbol{u}|_{\boldsymbol{H}^{1}(\Omega)}^{2}
$$

that is, $\|\boldsymbol{u}\|_{\boldsymbol{H}^{1}(\Omega)}+\|\operatorname{curl} \boldsymbol{H}\|_{\boldsymbol{L}^{2}(\Omega)} \leq C\|\boldsymbol{f}\|_{\boldsymbol{L}^{2}(\Omega)}$, where we have used Poincaré's inequality to $\boldsymbol{u}$. Moreover, applying Poincaré-type inequality to $\boldsymbol{H} \in \boldsymbol{W}(\operatorname{div} 0)$ and $\boldsymbol{A} \in \boldsymbol{D}(\operatorname{div} 0) \cap \boldsymbol{H}_{0}(\operatorname{div}, \Omega)$ (cf. e.g. [1]), we obtain

$$
\begin{aligned}
& \|\boldsymbol{H}\|_{\boldsymbol{H}(\operatorname{curl}, \Omega)} \leq C\left(\|\operatorname{curl} \boldsymbol{H}\|_{\boldsymbol{L}^{2}(\Omega)}+\|\operatorname{div} \boldsymbol{H}\|_{L^{2}(\Omega)}\right)=C\|\operatorname{curl} \boldsymbol{H}\|_{\boldsymbol{L}^{2}(\Omega)}, \\
& \|\boldsymbol{A}\|_{\boldsymbol{H}(\operatorname{curl}, \Omega)} \leq C\|\operatorname{curl} \boldsymbol{A}\|_{\boldsymbol{L}^{2}(\Omega)} \leq C\|\boldsymbol{H}\|_{\boldsymbol{L}^{2}(\Omega)} \leq C\|\operatorname{curl} \boldsymbol{H}\|_{\boldsymbol{L}^{2}(\Omega)} .
\end{aligned}
$$

The proof is completed.

\section{Mixed finite element approximation}

In this section, we study finite element approximation to the weak formulation of the MHD model. Inspired by [20, 24, the velocity $\boldsymbol{u}$ will be discretizd by $\boldsymbol{H}(\operatorname{div}, \Omega)$-conforming Brezzi-Douglas-Marini (BDM) elements and a DG-type formulation with interior penalties. Let $\mathcal{T}_{h}$ be a quasi-uniform and shaperegular tetrahedral mesh of $\Omega$. Let $h_{K}$ be the diameter of a tetrahedron $K \in \mathcal{T}_{h}$ and let $h=\max _{K \in \mathcal{T}_{h}} h_{K}$ denote the mesh size of $\mathcal{T}_{h}$.

\subsection{An interior-penalty finite element method}

First we introduce the finite element spaces for $\boldsymbol{u}, \boldsymbol{H}$, and $\boldsymbol{A}$ as follows

$$
\begin{aligned}
\boldsymbol{V}_{h} & :=\left\{\boldsymbol{v} \in \boldsymbol{H}_{0}(\operatorname{div}, \Omega):\left.\quad \boldsymbol{v}\right|_{K} \in \boldsymbol{P}_{1}(K), \quad \forall K \in \mathcal{T}_{h}\right\}, \\
\boldsymbol{W}_{h} & :=\left\{\boldsymbol{w} \in \boldsymbol{W}:\left.\boldsymbol{w}\right|_{K} \in \boldsymbol{P}_{1}(K), \quad \forall K \in \mathcal{T}_{h}\right\} \\
\boldsymbol{D}_{h} & :=\left\{\boldsymbol{d} \in \boldsymbol{D}:\left.\quad \boldsymbol{d}\right|_{K} \in \boldsymbol{P}_{1}(K), \quad \forall K \in \mathcal{T}_{h}\right\}
\end{aligned}
$$

where $\boldsymbol{P}_{k}(K)=\left(P_{k}(K)\right)^{3}$ and $P_{k}$ is the space of polynomials with degree $\leq k$. Functions in $\boldsymbol{V}_{h}$ are continuous normally but may be discontinuous tangentially, while functions in $\boldsymbol{W}_{h} \cup \boldsymbol{D}_{h}$ are continuous tangentially but may be discontinuous normally. The finite element spaces for multipliers $(p, r, \phi)$ are defined respectively by

$$
\begin{aligned}
Q_{h}:=\{q \in Q: & \left.\left.q\right|_{K} \in P_{0}(K), \quad \forall K \in \mathcal{T}_{h}\right\}, \\
S_{h}:=\{v \in S: & \left.\left.v\right|_{K} \in P_{2}(K), \quad \forall K \in \mathcal{T}_{h}\right\}, \\
Y_{h}:=\{\varphi \in Y: & \left.\left.\varphi\right|_{K} \in P_{2}(K), \quad \forall K \in \mathcal{T}_{h}\right\} .
\end{aligned}
$$

Clearly functions in $S_{h}$ and $Y_{h}$ are continuous.

Let $\mathcal{F}_{h}$ denote the set of faces of all tetrahedra in $\mathcal{T}_{h}$. We endow each $F \in \mathcal{F}_{h}$ with a unit normal $\boldsymbol{n}_{F}$ which points to the exterior of $\Omega$ when $F \subset \Gamma$ and to $K_{-}$when $F=\partial K_{+} \cap \partial K_{-}$for two adjacent elements $K_{ \pm} \in \mathcal{T}_{h}$. Let $\varphi$ be a scalar-, vectorial, or matrix-valued function which is piecewise smooth over $\mathcal{T}_{h}$. The mean value and the jump of $\varphi$ on $F$ are defined respectively by

$$
\{\{\varphi\}\}:=\left(\varphi_{+}+\varphi_{-}\right) / 2, \quad \llbracket \varphi \rrbracket:=\varphi_{+}-\varphi_{-} \quad \text { on } F,
$$

where $\varphi_{ \pm}$denote the traces of $\varphi$ on $F$ from inside of $K_{ \pm}$respectively. For any face $F=\partial K_{+} \cap \Gamma$, the mean value and the jump of $\varphi$ on $F$ are defined by

$$
\{\{\varphi\}\}=\llbracket \varphi \rrbracket=\varphi_{+} \quad \text { on } F .
$$


The discrete counterparts of $\mathscr{A}$ and $\mathscr{O}$ are defined by

$$
\begin{aligned}
\mathscr{A}_{h}(\boldsymbol{u}, \boldsymbol{v})= & \frac{1}{R_{e}} \sum_{K \in \mathcal{T}_{h}} \int_{K} \nabla \boldsymbol{u}: \nabla \boldsymbol{v}+\frac{\gamma}{R_{e}} \sum_{F \in \mathcal{F}_{h}} h_{F}^{-1} \int_{F} \llbracket \boldsymbol{u} \rrbracket \cdot \llbracket \boldsymbol{v} \rrbracket \\
& -\frac{1}{R_{e}} \sum_{F \in \mathcal{F}_{h}} \int_{F}\left(\left\{\left\{\frac{\partial \boldsymbol{u}}{\partial \boldsymbol{n}_{F}}\right\}\right\} \cdot \llbracket \boldsymbol{v} \rrbracket+\left\{\left\{\frac{\partial \boldsymbol{v}}{\partial \boldsymbol{n}_{F}}\right\}\right\} \cdot \llbracket \boldsymbol{u} \rrbracket\right), \\
\mathscr{O}_{h}(\boldsymbol{w} ; \boldsymbol{u}, \boldsymbol{v})= & -\sum_{K \in \mathcal{T}_{h}} \int_{K} \boldsymbol{u} \cdot \operatorname{div}(\boldsymbol{w} \otimes \boldsymbol{v})+\sum_{K \in \mathcal{T}_{h}} \int_{\partial K}\left(\boldsymbol{w} \cdot \boldsymbol{n}_{K}\right)\left(\boldsymbol{u}^{\downarrow} \cdot \boldsymbol{v}\right),
\end{aligned}
$$

where $\gamma>0$ is the penalty parameter, $\boldsymbol{n}_{K}$ the unit outer normal of $\partial K, h_{F}$ the diameter of $F$, and $\boldsymbol{u}^{\downarrow}$ the upwind convective flux defined by

$$
\boldsymbol{u}^{\downarrow}(\boldsymbol{x})= \begin{cases}\lim _{\epsilon \rightarrow 0^{+}} \boldsymbol{u}(\boldsymbol{x}-\epsilon \boldsymbol{w}(\boldsymbol{x})), & \boldsymbol{x} \in \partial K / \Gamma, \\ \mathbf{0}, & \boldsymbol{x} \in \partial K \cap \Gamma .\end{cases}
$$

In 16 , the second term in the right-hand side represents interior penalties used to insure the stability of discrete solutions.

The finite element approximation to problem $(14)$ is given as follows:

Find $\left(\boldsymbol{u}_{h}, \boldsymbol{H}_{h}, \boldsymbol{A}_{h}\right) \in \boldsymbol{V}_{h} \times \boldsymbol{W}_{h} \times \boldsymbol{D}_{h}$ and $\left(p_{h}, r_{h}, \phi_{h}\right) \in Q_{h} \times S_{h} \times Y_{h}$ such that

$$
\begin{aligned}
& \mathscr{A}_{h}\left(\boldsymbol{u}_{h}, \boldsymbol{v}_{h}\right)+\mathscr{O}_{h}\left(\boldsymbol{u}_{h} ; \boldsymbol{u}_{h}, \boldsymbol{v}_{h}\right)-\mathscr{L}\left(\boldsymbol{A}_{h} ; \boldsymbol{v}_{h}, \boldsymbol{H}_{h}\right)-\left(p_{h}, \operatorname{div} \boldsymbol{v}_{h}\right)=\left(\boldsymbol{f}, \boldsymbol{v}_{h}\right), \\
& \mathscr{C}_{1}\left(\boldsymbol{H}_{h}, \boldsymbol{w}_{h}\right)+\mathscr{L}\left(\boldsymbol{A}_{h} ; \boldsymbol{u}_{h}, \boldsymbol{w}_{h}\right)+\left(\nabla r_{h}, \boldsymbol{w}_{h}\right)=0, \\
& \mathscr{C}\left(\boldsymbol{A}_{h}, \boldsymbol{d}_{h}\right)-\left(\boldsymbol{H}_{h}, \operatorname{curl} \boldsymbol{d}_{h}\right)+\left(\nabla \phi_{h}, \boldsymbol{d}_{h}\right)=0, \\
& \left(\operatorname{div} \boldsymbol{u}_{h}, q_{h}\right)=0, \quad\left(\boldsymbol{H}_{h}, \nabla s_{h}\right)=0, \quad\left(\boldsymbol{A}_{h}, \nabla \varphi_{h}\right)=0,
\end{aligned}
$$

for all $\left(\boldsymbol{v}_{h}, \boldsymbol{w}_{h}, \boldsymbol{d}_{h}\right) \in \boldsymbol{V}_{h} \times \boldsymbol{W}_{h} \times \boldsymbol{D}_{h}$ and all $\left(q_{h}, s_{h}, \varphi_{h}\right) \in Q_{h} \times S_{h} \times Y_{h}$.

Due to $18 \mathrm{~d})$, we have $\left(\operatorname{div} \boldsymbol{u}_{h}, q_{h}\right)=0$ for any $q_{h} \in Q_{h}$. Because $\operatorname{div} \boldsymbol{u}_{h} \in Q_{h}$ also holds due to the definition of finite element space, thus we obtain

$$
\left(\operatorname{div} \boldsymbol{u}_{h}, \operatorname{div} \boldsymbol{u}_{h}\right)=0 \Rightarrow\left\|\operatorname{div} \boldsymbol{u}_{h}\right\|_{L^{2}}=0
$$

Because $\boldsymbol{u}_{h} \in \boldsymbol{H}(\operatorname{div}, \Omega)$, we have $\operatorname{div} \boldsymbol{u}_{h}=0$ exactly on the discrete level. This assertion directly comes from the work in [20. However, here we would like to mention that in the work [29], the $\boldsymbol{B}$ is also discretized by $\boldsymbol{H}(\operatorname{div}, \Omega)$-conforming element (Raviart-Thommas element there which is similar to BDM element) and one will only have $\left\|\operatorname{div} \boldsymbol{B}_{h}\right\|_{L^{2}}=0$ (see Lemma 2 of [29]). The authors in 29] claim that $\nabla \cdot \boldsymbol{B}_{h}=0$ holds exactly on the discrete level, so one can similar assertion that div $\boldsymbol{u}_{h}=0$ holds exactly. The main difference between our CT-FEM methods and the $\boldsymbol{B}-\boldsymbol{E}$ methods in 29$]$ is that we use magnetic potential $\boldsymbol{A}$ and magnetic field $\boldsymbol{H}$ as independent variables and therefore the divergence-free conditions for $\boldsymbol{J}$ and $\boldsymbol{B}$ in the Lorentz force are both satisfied naturally. Moreover as indicated by Theorem 4 in the following, our new finite element method is also energy stable.

We also remark that if $\mu$ is constant, the scheme 18 only enforces the constraint of $\boldsymbol{H}_{h}$ weakly. But our main concern is the divergence-free constraints in the momentum equations, especially in the Lorentz force $\boldsymbol{J}_{h} \times \boldsymbol{B}_{h}$, so we compromise and relax $\boldsymbol{H}_{h}$ 's constraint and just adopt edge element to discretize $\boldsymbol{H}_{h}$.

To make the $\boldsymbol{B}_{h}$ in the Lorentz force divergence-free, we recover a discrete magnetic vector potential $\boldsymbol{A}_{h}$ by solving a double curl problem using edge finite element method,

$$
\mathscr{C}\left(\boldsymbol{A}_{h}, \boldsymbol{d}_{h}\right)+\left(\nabla \phi_{h}, \boldsymbol{d}_{h}\right)=\left(\boldsymbol{H}_{h}, \operatorname{curl} \boldsymbol{d}_{h}\right), \quad\left(\boldsymbol{A}_{h}, \nabla \varphi_{h}\right)=0
$$

and then use $\boldsymbol{A}_{h}$ to compute discrete magnetic induction $\boldsymbol{B}_{h}=\operatorname{curl} \boldsymbol{A}_{h}$ in the Lorentz force. This philosophy has been used successfully in projection methods [6] and high-order CT methods on unstaggered grid [44. In [44, Section 4.2], for time-dependent ideal compressible MHD equaitons, Rossmanith solved the MHD equations using a "base scheme" to firstly obtain an intermediate magnetic field, which is not 
divergence-free. And then Rossmanith use the intermediate magnetic field to evolve the magnetic potentials to obtain the precisely divergence-free magnetic filed in the next time-step. We remark that $\boldsymbol{H}_{h}$ in our methods plays the same role as the pre-computed magnetic filed in Rossmanith's methods and other CT methods. The difference is that we solve a double curl problem instead to reconstruct the $\boldsymbol{B}_{h}$ in the Lorentz force. And this is the reason we call our methods by constrained transport divergence-free finite element.

\subsection{An iterative scheme for the discrete problem}

The discrete problem $(18)$ is a nonlinear system. Here we propose an iterative scheme of Picard type to solve the problem. Let $\boldsymbol{u}_{h}^{n-1} \in \boldsymbol{V}_{h}, \boldsymbol{A}_{h}^{n-1} \in \boldsymbol{D}_{h}, n \geq 1$, be the approximate solutions in the $(n-1)^{\text {th }}$ iteration. The approximate solutions in the $n^{\text {th }}$ iteration solve the coupled linear system:

Find $\left(\boldsymbol{u}_{h}^{n}, \boldsymbol{H}_{h}^{n}, \boldsymbol{A}_{h}^{n}\right) \in \boldsymbol{V}_{h} \times \boldsymbol{W}_{h} \times \boldsymbol{D}_{h}$ and $\left(p_{h}^{n}, r_{h}^{n}, \phi_{h}^{n}\right) \in Q_{h} \times S_{h} \times Y_{h}$ such that

$$
\begin{aligned}
& \mathscr{A}_{h}\left(\boldsymbol{u}_{h}^{n}, \boldsymbol{v}_{h}\right)+\mathscr{O}_{h}\left(\boldsymbol{u}_{h}^{n-1} ; \boldsymbol{u}_{h}^{n}, \boldsymbol{v}_{h}\right)-\mathscr{L}\left(\boldsymbol{A}_{h}^{n-1} ; \boldsymbol{v}_{h}, \boldsymbol{H}_{h}^{n}\right)-\left(p_{h}^{n}, \operatorname{div} \boldsymbol{v}_{h}\right)=\left(\boldsymbol{f}, \boldsymbol{v}_{h}\right), \\
& \mathscr{C}_{1}\left(\boldsymbol{H}_{h}^{n}, \boldsymbol{w}_{h}\right)+\mathscr{L}\left(\boldsymbol{A}_{h}^{n-1} ; \boldsymbol{u}_{h}^{n}, \boldsymbol{w}_{h}\right)+\left(\nabla r_{h}^{n}, \boldsymbol{w}_{h}\right)=0, \\
& \mathscr{C}\left(\boldsymbol{A}_{h}^{n}, \boldsymbol{d}_{h}\right)-\left(\boldsymbol{H}_{h}^{n}, \boldsymbol{c u r l} \boldsymbol{d}_{h}\right)+\left(\nabla \phi_{h}^{n}, \boldsymbol{d}_{h}\right)=0, \\
& \left(\operatorname{div} \boldsymbol{u}_{h}^{n}, q_{h}\right)=0, \quad\left(\boldsymbol{H}_{h}, \nabla s_{h}^{n}\right)=0, \quad\left(\boldsymbol{A}_{h}^{n}, \nabla \varphi_{h}\right)=0,
\end{aligned}
$$

for all $\left(\boldsymbol{v}_{h}, \boldsymbol{w}_{h}, \boldsymbol{d}_{h}\right) \in \boldsymbol{V}_{h} \times \boldsymbol{W}_{h} \times \boldsymbol{D}_{h}$ and all $\left(q_{h}, s_{h}, \varphi_{h}\right) \in Q_{h} \times S_{h} \times Y_{h}$.

The implicit upwind DG term $\mathscr{O}_{h}\left(\boldsymbol{w}_{h} ; \boldsymbol{u}_{h}, \boldsymbol{v}_{h}\right)$ is tailored to the standard convection term $\boldsymbol{w} \cdot \nabla \boldsymbol{u}$, which is difficult to modify for Newton's method. Also see Remark 3.3 of [20]. When upwinding is not used, Newtons method can be straightforwardly applied, which is just the case $\boldsymbol{P}_{2}-P_{1}$ Taylor-Hood element for $\boldsymbol{u}_{h}-p_{h}$ pair 32 . We admit that this is really a drawback for stationary problems where Newton's iteration may be more efficent nonlinear solver with relative large parameters (see numerical examples in 32]). The numerical experiments in the following also indicate that Picard iteration is not robust enough for large $R_{e}$ and $R_{m}$. In the future, acceleration techniques in optimization methods for nonlinear iteration can be incorporated for the present discretization.

\subsection{Well-posedness of 21}

First we introduce the discrete semi-norm and norm for piecewise regular functions

$$
|\varphi|_{1, h}:=\left(\sum_{K \in \mathcal{T}_{h}}|\varphi|_{H^{1}(K)}^{2}\right)^{1 / 2}, \quad\|\varphi\|_{1, h}:=\left(|\varphi|_{1, h}^{2}+\sum_{F \in \mathcal{F}_{h}} h_{F}^{-1} \int_{F} \llbracket \varphi \rrbracket^{2}\right)^{1 / 2} .
$$

The following lemma states that $\mathscr{A}_{h}$ is coercive and continuous and $\mathscr{O}_{h}$ is positive and continuous.

Lemma 3. 24, Lemma 3.2-3.3] Suppose $\gamma$ is large enough but independent of $h_{F}$ and $R_{e}$. There are constants $\theta_{1}, \theta_{2}>0$ independent of $h_{F}$ and $R_{e}$ such that

$$
\mathscr{A}_{h}(\boldsymbol{v}, \boldsymbol{v}) \geq \theta_{1} R_{e}^{-1}\|\boldsymbol{v}\|_{1, h}^{2}, \quad \mathscr{A}_{h}(\boldsymbol{u}, \boldsymbol{v}) \leq \theta_{2} R_{e}^{-1}\|\boldsymbol{u}\|_{1, h}\|\boldsymbol{v}\|_{1, h} \quad \forall \boldsymbol{u}, \boldsymbol{v} \in \boldsymbol{D}_{k}\left(\mathcal{T}_{h}\right),
$$

where $\boldsymbol{D}_{k}\left(\mathcal{T}_{h}\right)=\left\{\boldsymbol{v} \in \boldsymbol{L}^{2}(\Omega):\left.\boldsymbol{v}\right|_{K} \in \boldsymbol{P}_{k}(K), \forall K \in \mathcal{T}_{h}\right\}$. Moreover, there is a constant $C$ independent of $h$ such that, for any $\boldsymbol{w}, \boldsymbol{w}_{1} \in \boldsymbol{D}_{k}\left(\mathcal{T}_{h}\right) \cap \boldsymbol{H}(\operatorname{div} 0, \Omega)$,

$$
\begin{aligned}
& \mathscr{O}_{h}(\boldsymbol{w} ; \boldsymbol{v}, \boldsymbol{v})=\frac{1}{2} \sum_{F \in \mathcal{F}_{h}} \int_{F}|\boldsymbol{w} \cdot \boldsymbol{n}||\llbracket \boldsymbol{v} \rrbracket|^{2} \\
& \left|\mathscr{O}_{h}(\boldsymbol{w} ; \boldsymbol{u}, \boldsymbol{v})-\mathscr{O}_{h}\left(\boldsymbol{w}_{1} ; \boldsymbol{u}, \boldsymbol{v}\right)\right| \leq C\left\|\boldsymbol{w}-\boldsymbol{w}_{1}\right\|_{1, h}\|\boldsymbol{u}\|_{1, h}\|\boldsymbol{v}\|_{1, h} .
\end{aligned}
$$

For convenience, we define the divergence-free subspace of $\boldsymbol{V}_{h}$ by

$$
\boldsymbol{V}_{h}(\operatorname{div} 0)=\boldsymbol{V}_{h} \cap \boldsymbol{H}(\operatorname{div} 0, \Omega),
$$


and define the weakly divergence-free subspaces of $\boldsymbol{W}_{h}, \boldsymbol{D}_{h}$ by

$$
\begin{aligned}
\boldsymbol{W}_{h}(\operatorname{div} 0):=\left\{\boldsymbol{c}_{h} \in \boldsymbol{W}_{h}: \quad\left(\boldsymbol{c}_{h}, \nabla s_{h}\right)=0,\right. & \left.\forall s_{h} \in S_{h}\right\} \\
\boldsymbol{D}_{h}(\operatorname{div} 0): & =\left\{\boldsymbol{d}_{h} \in \boldsymbol{D}_{h}: \quad\left(\boldsymbol{d}_{h}, \nabla \varphi_{h}\right)=0, \quad \forall \varphi_{h} \in Y_{h}\right\}
\end{aligned}
$$

Generally, we have $\boldsymbol{W}_{h}(\operatorname{div} 0) \not \subset \boldsymbol{W}(\operatorname{div} 0)$ and $\boldsymbol{D}_{h}(\operatorname{div} 0) \not \subset \boldsymbol{D}(\operatorname{div} 0)$. However, the $\boldsymbol{L}^{2}(\Omega)$-orthogonal decompositions or discrete Helmholtz decompositions hold

$$
\boldsymbol{W}_{h}=\boldsymbol{W}_{h}(\operatorname{div} 0) \oplus \nabla S_{h}, \quad \boldsymbol{D}_{h}=\boldsymbol{D}_{h}(\operatorname{div} 0) \oplus \nabla Y_{h} .
$$

From [25. Theorem 4.7], there is a constant $C$ independent of $h$ such that the discrete Poincaré inequality holds

$$
\left\|\boldsymbol{c}_{h}\right\|_{\boldsymbol{L}^{2}(\Omega)} \leq C\left\|\operatorname{curl} \boldsymbol{c}_{h}\right\|_{\boldsymbol{L}^{2}(\Omega)} \quad \forall \boldsymbol{c}_{h} \in \boldsymbol{W}_{h}(\operatorname{div} 0) \cup \boldsymbol{D}_{h}(\operatorname{div} 0)
$$

From (21d), we easily find that

$$
\boldsymbol{u}_{h}^{n} \in \boldsymbol{V}_{h}(\operatorname{div} 0), \quad \boldsymbol{H}_{h}^{n} \in \boldsymbol{W}_{h}(\operatorname{div} 0), \quad \boldsymbol{A}_{h}^{n} \in \boldsymbol{D}_{h}(\operatorname{div} 0) .
$$

So (21) can be written into a reduced form: Find $\left(\boldsymbol{u}_{h}^{n}, \boldsymbol{H}_{h}^{n}\right) \in \boldsymbol{V}_{h}(\operatorname{div} 0) \times \boldsymbol{W}_{h}(\operatorname{div} 0)$ and $\boldsymbol{A}_{h}^{n} \in \boldsymbol{D}_{h}(\operatorname{div} 0)$ such that

$$
\begin{aligned}
& a\left(\left(\boldsymbol{u}_{h}^{n}, \boldsymbol{H}_{h}^{n}\right),\left(\boldsymbol{v}_{h}, \boldsymbol{w}_{h}\right)\right)=\left(\boldsymbol{f}, \boldsymbol{v}_{h}\right) \quad \forall\left(\boldsymbol{v}_{h}, \boldsymbol{w}_{h}\right) \in \boldsymbol{V}_{h}(\operatorname{div} 0) \times \boldsymbol{W}_{h}(\operatorname{div} 0), \\
& \mathscr{C}\left(\boldsymbol{A}_{h}^{n}, \boldsymbol{d}_{h}\right)=\left(\boldsymbol{H}_{h}^{n}, \operatorname{curl} \boldsymbol{d}_{h}\right) \quad \forall \boldsymbol{d}_{h} \in \boldsymbol{D}_{h}(\operatorname{div} 0),
\end{aligned}
$$

where the bilinear form $a$ is defined by

$$
\begin{aligned}
a\left(\left(\boldsymbol{u}_{h}^{n}, \boldsymbol{H}_{h}^{n}\right),\left(\boldsymbol{v}_{h}, \boldsymbol{w}_{h}\right)\right):= & \mathscr{A}_{h}\left(\boldsymbol{u}_{h}^{n}, \boldsymbol{v}_{h}\right)+\mathscr{O}_{h}\left(\boldsymbol{u}_{h}^{n-1} ; \boldsymbol{u}_{h}^{n}, \boldsymbol{v}_{h}\right)+\mathscr{C}_{1}\left(\boldsymbol{H}_{h}^{n}, \boldsymbol{w}_{h}\right) \\
& -\mathscr{L}\left(\boldsymbol{A}_{h}^{n-1} ; \boldsymbol{v}_{h}, \boldsymbol{H}_{h}^{n}\right)+\mathscr{L}\left(\boldsymbol{A}_{h}^{n-1} ; \boldsymbol{u}_{h}^{n}, \boldsymbol{w}_{h}\right) .
\end{aligned}
$$

Theorem 4. Problem (21) has unique solutions. There exists a constant $C$ independent of $h$ such that

$$
\left\|\boldsymbol{u}_{h}^{n}\right\|_{1, h}+\left\|\boldsymbol{H}_{h}^{n}\right\|_{\boldsymbol{H}(\operatorname{curl}, \Omega)}+\left\|\boldsymbol{A}_{h}^{n}\right\|_{\boldsymbol{H}(\operatorname{curl}, \Omega)} \leq C\|\boldsymbol{f}\|_{\boldsymbol{L}^{2}(\Omega)} .
$$

Proof. Since $\boldsymbol{u}_{n}^{n-1} \in \boldsymbol{V}_{h}(\operatorname{div} 0)$, we have $\operatorname{div} \boldsymbol{u}_{h}^{n-1}=0$. From Lemma 3 and inequality (23), it is easy to see that

$$
\begin{aligned}
a\left(\left(\boldsymbol{v}_{h}, \boldsymbol{w}_{h}\right),\left(\boldsymbol{v}_{h}, \boldsymbol{w}_{h}\right)\right) & \geq \mathscr{A}_{h}\left(\boldsymbol{v}_{h}, \boldsymbol{v}_{h}\right)+\kappa R_{m}^{-1}\left\|\operatorname{curl} \boldsymbol{w}_{h}\right\|_{\boldsymbol{L}^{2}(\Omega)}^{2} \\
& \geq C\left(\|\boldsymbol{v}\|_{1, h}^{2}+\left\|\boldsymbol{w}_{h}\right\|_{\boldsymbol{H}(\operatorname{curl}, \Omega)}\right)
\end{aligned}
$$

for all $\left(\boldsymbol{v}_{h}, \boldsymbol{w}_{h}\right) \in \boldsymbol{V}_{h}(\operatorname{div} 0) \times \boldsymbol{W}_{h}(\operatorname{div} 0)$, where $C>0$ is a constant independent of $h$. Therefore, the bilinear form $a$ is coercive on $\boldsymbol{V}_{h}(\operatorname{div} 0) \times \boldsymbol{W}_{h}(\operatorname{div} 0)$. So the finite dimensional problem 24a has unique solutions $\left(\boldsymbol{u}_{h}^{n}, \boldsymbol{H}_{h}^{n}\right)$. Moreover, 23$)$ implies that $\mathscr{C}$ is coercive on $\boldsymbol{D}_{h}(\operatorname{div} 0)$. So for the $\boldsymbol{H}_{h}^{n}$ obtained from 24a, problem 24b has a unique solution $\boldsymbol{A}_{h}^{n}$.

From [10, Proposition 3.3], there is a constant $C_{\mathrm{inf}}>0$ independent of $h$ such that

$$
\sup _{0 \neq \boldsymbol{v}_{h} \in \boldsymbol{V}_{h}} \frac{\left(\operatorname{div} \boldsymbol{v}_{h}, q_{h}\right)}{\left\|\boldsymbol{v}_{h}\right\|_{1, h}} \geq C_{\mathrm{inf}}\left\|q_{h}\right\|_{L^{2}(\Omega)} \quad \forall q_{h} \in Q_{h} .
$$

Moreover, by the inclusions $\nabla S_{h} \subset \boldsymbol{W}_{h}, \nabla Y_{h} \subset \boldsymbol{D}_{h}$ and Poincaré's inequality, there is a constant $\hat{C}_{\text {inf }}>0$ depending only on $\Omega$ such that

$$
\begin{aligned}
\sup _{0 \neq \boldsymbol{w}_{h} \in \boldsymbol{W}_{h}} \frac{\left(\boldsymbol{w}_{h}, \nabla s_{h}\right)}{\left\|\boldsymbol{w}_{h}\right\|_{\boldsymbol{H}(\mathbf{c u r l}, \Omega)}} \geq\left\|\nabla s_{h}\right\|_{\boldsymbol{L}^{2}(\Omega)} \geq \hat{C}_{\mathrm{inf}}\left\|s_{h}\right\|_{\boldsymbol{H}^{1}(\Omega)} & \forall s_{h} \in S_{h}, \\
\sup _{0 \neq \boldsymbol{d}_{h} \in \boldsymbol{D}_{h}} \frac{\left(\boldsymbol{d}_{h}, \nabla \varphi_{h}\right)}{\left\|\boldsymbol{d}_{h}\right\|_{\boldsymbol{H}(\operatorname{curl}, \Omega)}} \geq\left\|\nabla \varphi_{h}\right\|_{\boldsymbol{L}^{2}(\Omega)} \geq \hat{C}_{\mathrm{inf}}\left\|\varphi_{h}\right\|_{\boldsymbol{H}^{1}(\Omega)} & \forall \varphi_{h} \in Y_{h} .
\end{aligned}
$$

We conclude the existence and uniqueness of $p_{h}^{n}, r_{h}^{n}$, and $\phi_{h}^{n}$ from (21a) $\left.-21 \mathrm{c}\right)$.

Finally, the stability in (25) can be proven by arguments similar to the proof of Theorem 2 . We do not elaborate on the details. 
Since $\boldsymbol{V}_{h}, \boldsymbol{W}_{h}$, and $\boldsymbol{D}_{h}$ are finite dimensional, the stability 25 implies that, upon an extracted subsequence, the linearized solutions converge strongly to three functions $\boldsymbol{u}_{h} \in \boldsymbol{V}_{h}(\operatorname{div} 0), \boldsymbol{H}_{h} \in \boldsymbol{W}_{h}(\operatorname{div} 0)$, and $\boldsymbol{A}_{h} \in \boldsymbol{D}_{h}(\operatorname{div} 0)$. Moreover, the limits solve the nonlinear problem (18) and satisfy

$$
\left\|\boldsymbol{u}_{h}\right\|_{1, h}+\left\|\operatorname{curl} \boldsymbol{H}_{h}\right\|_{\boldsymbol{L}^{2}(\Omega)}+\left\|\operatorname{curl} \boldsymbol{A}_{h}\right\|_{\boldsymbol{L}^{2}(\Omega)} \leq C\|\boldsymbol{f}\|_{\boldsymbol{L}^{2}(\Omega)} .
$$

The convergence of the original sequence $\left\{\left(\boldsymbol{u}_{h}^{n}, \boldsymbol{H}_{h}^{n}, \boldsymbol{A}_{h}^{n}\right)\right\}$ and the uniqueness of solutions to (18) can be proven by arguments similar to [52] upon assuming that $R_{e}, R_{m}, \kappa$ are small enough. Since we are only interested in proposing the conservative scheme and its discrete solver, these are beyond the scope of this paper.

Remark 5. Because $\boldsymbol{H}_{h}$ and $\boldsymbol{A}_{h}$ belong to $\boldsymbol{H}(\mathbf{c u r l}, \Omega)$, we have $\boldsymbol{J}_{h}:=\operatorname{curl} \boldsymbol{H}_{h} \in \boldsymbol{H}(\operatorname{div}, \Omega)$ and $\boldsymbol{B}_{h}:=\operatorname{curl} \boldsymbol{A}_{h} \in \boldsymbol{H}(\operatorname{div}, \Omega)$ [35]. Thus the discrete current density and magnetic induction in the Lorentz force are precisely divergence-free because we can directly use divergence operator on $\boldsymbol{J}_{h}$ and $\boldsymbol{B}_{h}$. Moreover due to the mixed finite element for $\boldsymbol{u}_{h}-p_{h}$ with $\boldsymbol{H}$ (div)-conforming element for $\boldsymbol{u}_{h}$ the scheme is also mass-conservative (see [10, 30, 20] for details). Theorem 4 indicates that our finite element method is also energy stable.

\section{An augmented Lagrangian block preconditioner}

The purpose of this section is to propose a preconditioner for the linear algebraic systems resulting from the the Picard iteration (21). Because we use a monolithic way and the linear algebraic systems are a series of triple saddle-point problems, the usual Krylov subspace methods such as GMRES will be extremely slow to converge without preconditioning. And it would be ideal if the number of iterations using fixed tolerance did not grow under mesh refinement. Here we develop an augmented Lagrangian block preconditioner which follows the work in 32, where a grad-div stabilized formulation for the model in [52] is used. The basic ideas for approximate block factorization and operators' commutativity come from the work in 40, 41. Note that in our previous work 32] $\boldsymbol{P}_{2}-P_{1}$ Taylor-Hood Element is used for velocity-pressure pair. But now we adopt $\boldsymbol{H}(\operatorname{div}, \Omega)$-conforming element for velocity. To devise our preconditioner, we shall follow the approximate Schur complement techniques which has already gained much success in incompressible Navier-Stokes equations [13].

\subsection{Algebraic form of problem (21)}

Since $\operatorname{div} \boldsymbol{u}_{h}^{n}=0$, we add a grad-div stabilization term in the momentum equation and rewrite (21) as follows

$$
\begin{aligned}
& \mathscr{C}\left(\boldsymbol{A}_{h}^{n}, \boldsymbol{d}_{h}\right)-\left(\boldsymbol{H}_{h}^{n}, \operatorname{curl} \boldsymbol{d}_{h}\right)+\left(\nabla \phi_{h}^{n}, \boldsymbol{d}_{h}\right)=0, \\
& \left(\boldsymbol{A}_{h}^{n}, \nabla \varphi_{h}\right)=0, \\
& \mathscr{C}_{1}\left(\boldsymbol{H}_{h}^{n}, \boldsymbol{w}_{h}\right)+\mathscr{L}\left(\boldsymbol{A}_{h}^{n-1} ; \boldsymbol{u}_{h}^{n}, \boldsymbol{w}_{h}\right)+\left(\nabla r_{h}^{n}, \boldsymbol{w}_{h}\right)=0, \\
& \left(\boldsymbol{H}_{h}, \nabla s_{h}^{n}\right)=0, \\
& \mathscr{A}_{1}\left(\boldsymbol{u}_{h}^{n}, \boldsymbol{v}_{h}\right)+\mathscr{O}_{h}\left(\boldsymbol{u}_{h}^{n-1} ; \boldsymbol{u}_{h}^{n}, \boldsymbol{v}_{h}\right)-\mathscr{L}\left(\boldsymbol{A}_{h}^{n-1} ; \boldsymbol{v}_{h}, \boldsymbol{H}_{h}^{n}\right)-\left(p_{h}^{n}, \operatorname{div} \boldsymbol{v}_{h}\right)=\left(\boldsymbol{f}, \boldsymbol{v}_{h}\right), \\
& \left(\operatorname{div} \boldsymbol{u}_{h}^{n}, q_{h}\right)=0,
\end{aligned}
$$

for all $\left(\boldsymbol{v}_{h}, \boldsymbol{w}_{h}, \boldsymbol{d}_{h}\right) \in \boldsymbol{V}_{h} \times \boldsymbol{W}_{h} \times \boldsymbol{D}_{h}$ and all $\left(q_{h}, s_{h}, \varphi_{h}\right) \in Q_{h} \times S_{h} \times Y_{h}$, where

$$
\mathscr{A}_{1}\left(\boldsymbol{w}_{h}, \boldsymbol{v}_{h}\right):=\mathscr{A}_{h}\left(\boldsymbol{w}_{h}, \boldsymbol{v}_{h}\right)+\alpha\left(\operatorname{div} \boldsymbol{w}_{h}, \operatorname{div} \boldsymbol{v}_{h}\right)
$$

We have rearranged the variables order as $\left(\boldsymbol{A}_{h}, \phi_{h}, \boldsymbol{H}_{h}, r_{h}, \boldsymbol{u}_{h}, p_{h}\right)$ for easy block factorization and preconditioning. The adding term $\alpha\left(\operatorname{div} \boldsymbol{u}_{n}, \operatorname{div} \boldsymbol{v}\right)$ is called grad-div stabilization 39 and $\alpha$ is called the grad-div parameter. Because div $\boldsymbol{u}_{h}=0$ can be guaranteed [20, it does not change the discrete solutions compared with that using Taylor-Hood element, but enhances the performance of the preconditioner. Here we reiterate that though we realize the three divergence-free constraints for the momentum equation, it is at the expense of more degrees of freedom because we add variables $\boldsymbol{A}$ and $\phi$. The new CT formulation can also be regarded as a modification of the model in 20, namely an extra double curl problem for $\boldsymbol{A}$ is solved to ensure the divergence-free $\boldsymbol{B}_{h}$ in the Lorentz force. This fact means that for the proposed scheme existing FEM codes can be reused in a simple way other than a classical double curl problem solve for magnetic vector potential. 
The linear system can be written into an algebraic form

$$
\mathbb{A} \mathbf{x}=\mathbf{b},
$$

where $\mathbb{A}$ is the stiffness matrix, $\mathbf{x}$ the vector of DOFs, and $\mathbf{b}$ the load vector. They are given in block forms by

$$
\mathbb{A}=\left(\begin{array}{cccccc}
\mathbb{C} & \mathbb{G}^{\top} & \mathbb{K} & 0 & 0 & 0 \\
\mathbb{G} & 0 & 0 & 0 & 0 & 0 \\
0 & 0 & \mathbb{H} & \mathbb{D}^{\top} & \mathbb{J}^{\top} & 0 \\
0 & 0 & \mathbb{D} & 0 & 0 & 0 \\
0 & 0 & -\mathbb{J} & 0 & \mathbb{F} & \mathbb{B}^{\top} \\
0 & 0 & 0 & 0 & \mathbb{B} & 0
\end{array}\right), \quad \mathbf{x}=\left(\begin{array}{c}
\mathbf{x}_{A} \\
\mathbf{x}_{\phi} \\
\mathbf{x}_{H} \\
\mathbf{x}_{r} \\
\mathbf{x}_{u} \\
\mathbf{x}_{p}
\end{array}\right), \quad \mathbf{b}=\left(\begin{array}{c}
\mathbf{b}_{A} \\
\mathbf{b}_{\phi} \\
\mathbf{b}_{H} \\
\mathbf{b}_{r} \\
\mathbf{b}_{u} \\
\mathbf{b}_{p}
\end{array}\right)
$$

Here $\mathbf{x}_{A}, \mathbf{x}_{\phi}, \mathbf{x}_{H}, \mathbf{x}_{r}, \mathbf{x}_{u}, \mathbf{x}_{p}$ are vectors of DOFs belonging to $\boldsymbol{A}_{n}, \phi_{n}, \boldsymbol{H}_{n}, r_{n}, \boldsymbol{u}_{n}, p_{n}$ respectively and $\mathbf{b}_{A}, \mathbf{b}_{\phi}, \mathbf{b}_{H}, \mathbf{b}_{r}, \mathbf{b}_{u}, \mathbf{b}_{p}$ are the corresponding load vectors. The block matrices $\mathbb{C}, \mathbb{G}, \mathbb{K}, \mathbb{H}, \mathbb{D}, \mathbb{J}, \mathbb{F}$ and $\mathbb{B}$ are Galerkin matrices defined by

$$
\begin{array}{lll}
\mathbb{C} \leftrightarrow \mathscr{C}\left(\boldsymbol{A}_{h}^{n}, \boldsymbol{d}_{h}\right), \quad \mathbb{G} \leftrightarrow\left(\boldsymbol{A}_{h}^{n}, \nabla \varphi_{h}\right), & \mathbb{K} \leftrightarrow-\left(\boldsymbol{H}_{h}^{n}, \operatorname{curl} \boldsymbol{d}_{h}\right), \\
\mathbb{H} \leftrightarrow \mathscr{C}_{1}\left(\boldsymbol{H}_{h}^{n}, \boldsymbol{d}_{h}\right), \quad \mathbb{D} \leftrightarrow\left(\boldsymbol{H}_{h}^{n}, \nabla s_{h}\right), & \mathbb{J} \leftrightarrow \mathscr{L}\left(\boldsymbol{A}_{h}^{n-1} ; \boldsymbol{v}_{h}, \boldsymbol{H}_{h}^{n}\right), \\
\mathbb{F} \leftrightarrow \mathscr{A}_{1}\left(\boldsymbol{u}_{h}^{n}, \boldsymbol{v}_{h}\right)+\mathscr{O}_{h}\left(\boldsymbol{u}_{h}^{n-1} ; \boldsymbol{u}_{h}^{n}, \boldsymbol{v}_{h}\right), & \mathbb{B} \leftrightarrow-\left(p_{h}^{n}, \operatorname{div} \boldsymbol{v}_{h}\right) .
\end{array}
$$

We remark that compared with the formulation in 20, 41, 32, the extra cost for the linear algebraic equations is only the classical double curl saddle problem for the magnetic vector potential $\boldsymbol{A}_{h}$

$$
\left(\begin{array}{cc}
\mathbb{C} & \mathbb{G}^{\top} \\
\mathbb{G} & 0
\end{array}\right)
$$

\subsection{Ideal preconditioner of $\mathbb{A}$}

Now we deduce a preconditioner of $\mathbb{A}$ based on LU factorization and approximate Schur complements. Since $\mathbb{C}$ and $\mathbb{H}$ are discretization of curl curl operators, they are singular matrices. We first apply mass augmentation techniques to the two saddle structures for $\mathbb{C}$ and $\mathbb{H}$ (see more details in 21. for the augmentation of Maxwell saddle-point problem and Section 3.1 of [41] for MHD)

$$
\left(\begin{array}{cc}
\mathbb{C} & \mathbb{G}^{\top} \\
\mathbb{G} & 0
\end{array}\right), \quad\left(\begin{array}{cc}
\mathbb{H} & \mathbb{D}^{\top} \\
\mathbb{D} & 0
\end{array}\right) .
$$

One will obtain the following factorization $\mathbb{A}=\mathbb{E} \tilde{\mathbb{A}}$ where

$$
\begin{aligned}
\mathbb{E} & =\left(\begin{array}{cccccc}
\mathbb{I}_{A} & -\mathbb{G}^{\top} \mathbb{L}_{\phi}^{-1} & 0 & 0 & 0 & 0 \\
0 & \mathbb{I}_{\phi} & 0 & 0 & 0 & 0 \\
0 & 0 & \mathbb{I}_{H} & -\mathbb{D}^{\top} \mathbb{L}_{r}^{-1} & 0 & 0 \\
0 & 0 & 0 & \mathbb{I}_{r} & 0 & 0 \\
0 & 0 & 0 & 0 & \mathbb{I}_{u} & 0 \\
0 & 0 & 0 & 0 & 0 & \mathbb{I}_{p}
\end{array}\right), \\
\tilde{\mathbb{A}} & =\left(\begin{array}{cccccc}
\tilde{\mathbb{C}} & \mathbb{G}^{\top} & \mathbb{K} & 0 & 0 & 0 \\
\mathbb{G} & 0 & 0 & 0 & 0 & 0 \\
0 & 0 & \tilde{\mathbb{H}} & \mathbb{D}^{\top} & \mathbb{J}^{\top} & 0 \\
0 & 0 & \mathbb{D} & 0 & 0 & 0 \\
0 & 0 & -\mathbb{J} & 0 & \mathbb{F} & \mathbb{B}^{\top} \\
0 & 0 & 0 & 0 & \mathbb{B} & 0
\end{array}\right) .
\end{aligned}
$$

The diagonal block matrices of $\mathbb{E}$ are all identity matrices of different sizes, $\mathbb{L}_{\phi}$ is the stiffness matrix of $-\Delta$ on $Y_{h}, \mathbb{L}_{r}$ is the stiffness matrix of $-\left(R_{m} / \kappa\right) \Delta$ on $S_{h}$, and

$$
\tilde{\mathbb{C}}=\mathbb{C}+\mathbb{G}^{\top} \mathbb{L}_{\phi}^{-1} \mathbb{G}, \quad \tilde{\mathbb{H}}=\mathbb{H}+\mathbb{D}^{\top} \mathbb{L}_{r}^{-1} \mathbb{D} .
$$


$\widetilde{\mathbb{C}}$ and $\tilde{\mathbb{H}}$ are called mass augmentation of $\mathbb{C}$ and $\mathbb{H}$ because we can use mass matrices to approximate $\mathbb{G}^{\top} \mathbb{L}_{\phi}^{-1} \mathbb{G}$ and $\mathbb{D}^{\top} \mathbb{L}_{r}^{-1} \mathbb{D}[21,41]$. We further consider the LU factorization $\tilde{\mathbb{A}}=\mathbb{L} \mathbb{U}$ where

$$
\begin{aligned}
& \mathbb{L}=\left(\begin{array}{cccccc}
\mathbb{I}_{A} & 0 & 0 & 0 & 0 & 0 \\
\mathbb{G} \tilde{\mathbb{C}}^{-1} & \mathbb{I} & 0 & 0 & 0 & 0 \\
0 & 0 & \mathbb{I}_{\phi} & 0 & 0 & 0 \\
0 & 0 & \mathbb{D} \tilde{H}^{-1} & \mathbb{I}_{H} & 0 & 0 \\
0 & 0 & -\mathbb{J} \tilde{\mathbb{H}}^{-1} & -\mathbb{J U H}^{-1} \mathbb{D}^{\top} \mathbb{S}_{r}^{-1} & \mathbb{I}_{u} & 0 \\
0 & 0 & 0 & 0 & \mathbb{B}^{-1} & \mathbb{I}_{p}
\end{array}\right), \\
& \mathbb{U}=\left(\begin{array}{cccccc}
\tilde{\mathbb{C}} & \mathbb{G}^{\top} & \mathbb{K} & 0 & 0 & 0 \\
0 & -\mathbb{S}_{\phi} & -\mathbb{G} \tilde{\mathbb{C}}^{-1} \mathbb{K} & 0 & 0 & 0 \\
0 & 0 & \tilde{\mathbb{H}} & \mathbb{D}^{\top} & \mathbb{J}^{\top} & 0 \\
0 & 0 & 0 & -\mathbb{S}_{r} & -\tilde{D}_{\mathbb{H}}^{-1} \mathbb{J}^{\top} & 0 \\
0 & 0 & 0 & 0 & \tilde{\mathbb{F}} & \mathbb{B}^{\top} \\
0 & 0 & 0 & 0 & 0 & -\mathbb{S}_{p}
\end{array}\right) .
\end{aligned}
$$

The diagonal block matrices of $\mathbb{U}$ are given by

$$
\begin{aligned}
& \mathbb{S}_{\phi}=\mathbb{G} \tilde{\mathbb{C}}^{-1} \mathbb{G}^{\top}, \quad \mathbb{S}_{r}=\mathbb{D} \tilde{\mathbb{H}}^{-1} \mathbb{D}^{\top}, \quad \mathbb{S}_{p}=\mathbb{B}^{-1} \mathbb{B}^{\top}, \\
& \tilde{\mathbb{F}}:=\mathbb{F}+\mathbb{J} \tilde{\mathbb{H}}^{-1} \mathbb{J}^{\top}-\left(\mathbb{D} \tilde{H}^{-1} \mathbb{J}^{\top}\right)^{\top} \mathbb{S}_{r}^{-1}\left(\mathbb{D} \tilde{H}^{-1} \mathbb{J}^{\top}\right) .
\end{aligned}
$$

It is easy to see that $\mathbb{A}(\mathbb{E} \mathbb{U})^{-1}=\mathbb{E} \mathbb{L} \mathbb{E}^{-1}$. Since $\mathbb{L}$ only has unit eigenvalues, we call $(\mathbb{E} \mathbb{U})^{-1}$ an ideal preconditioner of $\mathbb{A}$.

Direct calculations show

$$
\mathbb{E} \mathbb{U}=\left(\begin{array}{cccccc}
\tilde{\mathbb{C}} & \mathbb{X}_{12} & \mathbb{X}_{13} & 0 & 0 & 0 \\
0 & -\mathbb{S}_{\phi} & \mathbb{X}_{23} & 0 & 0 & 0 \\
0 & 0 & \widetilde{\mathbb{H}} & \mathbb{X}_{34} & \mathbb{X}_{35} & 0 \\
0 & 0 & 0 & -\mathbb{S}_{r} & \mathbb{X}_{45} & 0 \\
0 & 0 & 0 & 0 & \tilde{\mathbb{F}} & \mathbb{B}^{\top} \\
0 & 0 & 0 & 0 & 0 & -\mathbb{S}_{p}
\end{array}\right)
$$

where

$$
\begin{array}{lll}
\mathbb{X}_{12}=\mathbb{G}^{\top}+\mathbb{G}^{\top} \mathbb{L}_{\phi}^{-1} \mathbb{S}_{\phi}, & \mathbb{X}_{13}=\mathbb{K}-\mathbb{G}^{\top} \mathbb{L}_{\phi}^{-1} \mathbb{X}_{23}, & \mathbb{X}_{23}=-\mathbb{G} \tilde{\mathbb{C}}^{-1} \mathbb{K} \\
\mathbb{X}_{34}=\mathbb{D}^{\top}+\mathbb{D}^{\top} \mathbb{L}_{r}^{-1} \mathbb{S}_{r}, & \mathbb{X}_{35}=\mathbb{J}^{\top}-\mathbb{D}^{\top} \mathbb{L}_{r}^{-1} \mathbb{X}_{45}, & \mathbb{X}_{45}=-\mathbb{D} \tilde{H}^{-1} \mathbb{J}^{\top}
\end{array}
$$

\subsection{Practical preconditioner of $\mathbb{A}$}

The block entries of $\mathbb{E} \mathbb{U}$ is too complex to provide a practical preconditioner. We simplify them based on heuristic analysis.

Remember that the block matrices of $\mathbb{A}$ are algebraic representations of differential operators or multiplication operators appearing in the MHD system, e.g.,

$$
\tilde{\mathbb{C}} \Leftrightarrow \text { curl curl }+\nabla\left(-\left.\Delta\right|_{Y_{h}}\right)^{-1}(-\operatorname{div}), \quad \mathbb{G} \Leftrightarrow-\operatorname{div} \quad \text { on } \boldsymbol{D}_{h},
$$

where (-div) is understood as the dual operator of $\left.\nabla\right|_{Y_{h}}$. Similarly

$$
\tilde{\mathbb{H}} \Leftrightarrow \kappa R_{m}^{-1}\left[\operatorname{curl} \operatorname{curl}+\nabla\left(-\left.\Delta\right|_{S_{h}}\right)^{-1}(-\operatorname{div})\right], \quad \mathbb{D} \Leftrightarrow-\operatorname{div} \quad \text { on } \boldsymbol{W}_{h},
$$

where (-div) is understood as the dual operator of $\left.\nabla\right|_{S_{h}}$. From [21, we have the following spectral equivalences of matrices

$$
\tilde{\mathbb{C}} \sim \hat{\mathbb{C}}:=\mathbb{C}+\mathbb{M}_{A}, \quad \tilde{\mathbb{H}} \sim \hat{\mathbb{H}}:=\mathbb{H}+\kappa R_{m}^{-1} \mathbb{M}_{H}, \quad \mathbb{S}_{\phi} \sim \mathbb{L}_{\phi}, \quad \mathbb{S}_{r} \sim \mathbb{L}_{r},
$$

where $\mathbb{M}_{A}, \mathbb{M}_{H}$ are mass matrices on $\boldsymbol{D}_{h}$ and $\boldsymbol{W}_{h}$ respectively. This inspires us to make the replacements for off-diagonal blocks of $\mathbb{E} \mathbb{U}$

$$
\mathbb{X}_{12} \approx 2 \mathbb{G}^{\top}, \quad \mathbb{X}_{34} \approx 2 \mathbb{D}^{\top} .
$$


Now we shall follow the arguments of 32 to estimate $\mathbb{X}_{23}$ and $\mathbb{X}_{45}$. Note that $\mathbb{K}$ and $\mathbb{J}$ are algebraic representations of two multiplication operators

$$
\mathbb{K} \Leftrightarrow\left(-\operatorname{curl} \boldsymbol{H}_{h}^{n}\right) \quad \text { on } \quad \boldsymbol{D}_{h}, \quad \mathbb{J}^{\top} \Leftrightarrow \kappa \operatorname{curl}\left(\operatorname{curl} \boldsymbol{A}_{h}^{n-1} \times \boldsymbol{u}_{h}^{n}\right) \quad \text { on } \boldsymbol{W}_{h} .
$$

Formally we have the identity $(-\operatorname{div})\left(\right.$ curlcurl $\left.+\nabla \Delta^{-1} \operatorname{div}\right)=-\operatorname{div}$ on $C_{0}^{\infty}(\Omega)$. This shows that $(-\operatorname{div})\left(\operatorname{curl} \operatorname{curl}+\nabla \Delta^{-1} \operatorname{div}\right)^{-1}=-\operatorname{div}$ on $\boldsymbol{C}_{0}^{\infty}$. Now from (28) and (32), we deduce heuristically that

$$
\mathbb{X}_{23}=-\mathbb{G} \tilde{\mathbb{C}}^{-1} \mathbb{K} \approx-\mathbb{G} \mathbb{K} \approx 0 .
$$

Similarly, from 28$)$ and $(32)$, we deduce heuristically that

$$
\mathbb{X}_{45}=-\mathbb{D} \tilde{H}^{-1} \mathbb{J}^{\top} \approx-\mathbb{D}^{\top} \approx 0 .
$$

Based on (33) and (34), we also have the approximations

$$
\mathbb{X}_{13} \approx \mathbb{K}, \quad \mathbb{X}_{35} \approx \mathbb{J}^{\top} .
$$

Now it is left to consider the Navier-Stokes block of $\mathbb{E} \mathbb{U}$. From (30) and (34), we have the following approximations

$$
\tilde{\mathbb{F}} \approx \mathbb{F}+\mathbb{\mathbb { H }} \tilde{\mathbb{H}}^{-1} \mathbb{J}^{\top} \approx \mathbb{F}+\mathbb{J} \hat{H}^{-1} \mathbb{J}^{\top}, \quad \mathbb{S}_{p}=\mathbb{B}^{-1} \mathbb{B}^{\top} \approx \mathbb{B}\left(\mathbb{F}+\mathbb{J} \hat{H}^{-1} \mathbb{J}^{\top}\right)^{-1} \mathbb{B}^{\top} .
$$

Here $\int \hat{H}^{-1} \mathbb{J}^{\top}$ stands for coupling term between the fluid and electromagnetic field. In [32], Li and Zheng derived approximate Schur complements for $\mathbb{F}+\mathbb{J} \hat{H}^{-1} \mathbb{J}^{\top}$ and $\mathbb{S}_{p}$ in the case that the Navier-Stokes equations are solved by $\boldsymbol{P}_{2}-P_{1}$ Taylor-Hood finite elements. Here we apply their results directly to our case of $\left(\boldsymbol{u}_{h}^{n}, p_{h}^{n}\right) \in \boldsymbol{V}_{h} \times Q_{h}$. In [32, Section 3.3], Li and Zheng suggested to approximate $\mathbb{F}+\mathbb{J} \hat{H}^{-1} \mathbb{J}^{\top}$ and $\mathbb{S}_{p}$ as follows

$$
\tilde{\mathbb{F}} \approx \mathbb{F}+\mathbb{J} \hat{\mathbb{H}}^{-1} \mathbb{J}^{\top} \approx \mathbb{S}_{u}, \quad \mathbb{S}_{p} \approx \mathbb{B S}_{u}^{-1} \mathbb{B}^{\top} \approx\left(R_{e}^{-1}+\alpha\right)^{-1} \mathbb{M}_{p}
$$

where $\mathbb{S}_{u}$ is the stiffness matrix associated with the bilinear form

$$
\mathscr{A}_{u}(\boldsymbol{w}, \boldsymbol{v}):=\mathscr{A}_{1}(\boldsymbol{w}, \boldsymbol{v})+\mathscr{O}_{h}\left(\boldsymbol{u}_{h}^{n-1} ; \boldsymbol{w}, \boldsymbol{v}\right)+\kappa R_{m}\left(\operatorname{curl} \boldsymbol{A}_{h}^{n-1} \times \boldsymbol{w}, \operatorname{curl} \boldsymbol{A}_{h}^{n-1} \times \boldsymbol{v}\right),
$$

which means that we use $\kappa R_{m}\left(\operatorname{curl} \boldsymbol{A}_{h}^{n-1} \times \boldsymbol{w}, \operatorname{curl} \boldsymbol{A}_{h}^{n-1} \times \boldsymbol{v}\right)$ to approximate $\mathbb{J} \hat{\mathbb{H}}^{-1} \mathbb{J}^{\top} 32$. Here $\mathbb{M}_{p}$ is the mass Matrix on finite element space $Q_{h}$, and $\alpha$ is the grad-div stabilization parameter. We refer to [3, 4, 5, 15] for more details about augmented Lagrangian preconditioners for solving Navier-Stokes equations.

Finally, using the approximations $\sqrt{30-(36)}$ in $\mathbb{E} \mathbb{U}$, a practical preconditioner of $\mathbb{A}$ can be defined by the inverse of

$$
\mathbb{P}=\left(\begin{array}{cccccc}
\hat{\mathbb{C}} & 2 \mathbb{G}^{\top} & \mathbb{K} & 0 & 0 & 0 \\
0 & -\mathbb{L}_{\phi} & 0 & 0 & 0 & 0 \\
0 & 0 & \hat{\mathbb{H}} & 2 \mathbb{D}^{\top} & \mathbb{J}^{\top} & 0 \\
0 & 0 & 0 & -\mathbb{L}_{r} & 0 & 0 \\
0 & 0 & 0 & 0 & \mathbb{S}_{u} & \mathbb{B}^{\top} \\
0 & 0 & 0 & 0 & 0 & -\left(R_{e}^{-1}+\alpha\right)^{-1} \mathbb{M}_{p}
\end{array}\right)
$$

\subsection{A preconditioned GMRES algorithm}

Based on $\mathbb{P}$, we propose a preconditioned GMRES method for solving (26). In each GMRES iteration, one needs to solve the system of algebraic equations

$$
\mathbb{P e}=\mathbf{r}
$$

where $\mathbf{e}$ is the correction vector and $\mathbf{r}$ the residual vector calculated from last iteration. Now we present the algorithm for solving an approximate solution of 38 .

Algorithm 6. Set the tolerance $\varepsilon_{0}=10^{-3}$ and write

$$
\mathbf{e}=\left(\mathbf{e}_{A}, \mathbf{e}_{\phi}, \mathbf{e}_{H}, \mathbf{e}_{r}, \mathbf{e}_{u}, \mathbf{e}_{p}\right)^{\top}, \quad \mathbf{r}=\left(\mathbf{r}_{A}, \mathbf{r}_{\phi}, \mathbf{r}_{H}, \mathbf{r}_{r}, \mathbf{r}_{u}, \mathbf{r}_{p}\right)^{\top}
$$

The approximate solution of 38 is computed in six steps below. In each step, the algebraic problem is solved iteratively until the relative residual is less than $\varepsilon_{0}$. 
1. Solve $\mathbb{M}_{p} \mathbf{e}_{p}=-\left(R_{e}^{-1}+\alpha\right) \mathbf{r}_{p}$ by the $\mathrm{CG}$ method with diagonal preconditioner.

2. Solve $\mathbb{S}_{u} \mathbf{e}_{u}=\mathbf{r}_{u}-\mathbb{B}^{\top} \mathbf{e}_{p}$ by the GMRES method with additive Schwarz preconditioner (cf. [11]).

3. Solve $\mathbb{L}_{r} \mathbf{e}_{r}=-\mathbf{r}_{r}$ by the CG method with algebraic multigrid solver (cf. [28]).

4. Solve $\hat{\mathbb{H}} \mathbf{e}_{H}=\mathbf{r}_{H}-2 \mathbb{D}^{\top} \mathbf{e}_{r}-\mathbb{J}^{\top} \mathbf{e}_{u}$ by the CG method with auxiliary space preconditioner (cf. [26]).

5. Solve $\mathbb{L}_{\phi} \mathbf{e}_{\phi}=-\mathbf{r}_{\phi}$ by the CG method with algebraic multigrid solver.

6. Solve $\hat{\mathbb{C}} \mathbf{e}_{A}=\mathbf{r}_{A}-2 \mathbb{G}^{\top} \mathbf{e}_{\phi}-\mathbb{K} \mathbf{e}_{H}$ by the CG method with auxiliary space preconditioner.

We remark that the total number of iterations for solving $\mathbb{A} \mathbf{x}=\mathbf{b}$ is insensitive to the choice of tolerance $\varepsilon_{0}$ in Algorithm 6 when $\varepsilon_{0} \leq 10^{-2}$. We should confess that the additive Schwarz preconditioner adopted in Step 2 of Algorithm 6 is not optimal. More efficient preconditioners for $\mathbb{S}_{u}$ are important to improve the overall efficiency and will be our future work. We refer the readers to recent work 15 , on threedimensional stationary Navier-Stokes equations using augmented Lagrangian block preconitioner, where an optimal geometrical multigrid method is developed. However an extension of the multigrid techniques for the solving of the Step 2 problem, where $\boldsymbol{H}$ (div)-conforming element is used for the velocity field $\boldsymbol{u}_{h}$, is not a trivial thing.

\section{$5 \quad$ Numerical experiments}

In this section, we report several numerical experiments to show convergence orders of discrete solutions and to demonstrate the performance of the preconditioned GMRES solver. The finite element method and the discrete solver are implemented on the finite element package "Parallel Hierarchical Grid" (PHG) [55].

For solving the nonlinear problem (18), the Picard iterations stop whenever the criterion is reached

$$
\Theta\left(\boldsymbol{u}_{h}^{n}\right)+\Theta\left(\boldsymbol{H}_{h}^{n}\right)+\Theta\left(\boldsymbol{A}_{h}^{n}\right)<\delta,
$$

where $\Theta\left(\boldsymbol{w}_{h}^{n}\right)=\left\|\boldsymbol{w}_{h}^{n}-\boldsymbol{w}_{h}^{n-1}\right\|_{\boldsymbol{L}^{2}(\Omega)}\left\|\boldsymbol{w}_{h}^{n}\right\|_{\boldsymbol{L}^{2}(\Omega)}^{-1}$ for $\boldsymbol{w}_{h}^{n}=\boldsymbol{u}_{h}^{n}, \boldsymbol{H}_{h}^{n}$, and $\boldsymbol{A}_{h}^{n}$. Here $\delta$ is the tolerance for Picard's iterations. For solving the linear problem (26), let $\mathbf{x}^{(k)}, k \geq 0$, be the approximate solution at $k^{\text {th }}$ GMRES iteration and let $\mathbf{r}^{(k)}=\mathbf{b}-\mathbb{A} \mathbf{x}^{(k)}$ be the residual. The iterations stop whenever the criterion is reached

$$
\left\|\mathbf{r}^{(k)}\right\|_{2} \leq \varepsilon\left\|\mathbf{r}^{(0)}\right\|_{2}
$$

where $\varepsilon$ is the tolerance for the GMRES solver. The maximal iteration number for the GMRES solver is set by 200 without restart and right preconditioning algorithm is adopted here.

Throughout this section, we set the penalty parameter in 116 by $\gamma=10$ and the grad-div parameter in $\mathscr{A}_{1}$ by $\alpha=1$, except for Example 5.4 where the sensitivity of the solver to $\alpha$ is tested. The domain is chosen as $\Omega=[0,1]^{3}$. We choose 5 quasi-uniform meshes of $\Omega$ by successive refinements. The information of the meshes is listed in Table 1

Table 1: Five successively refined meshes.

\begin{tabular}{|c|c|r|r|r|}
\hline Mesh & $h$ & DOFs for $\left(\boldsymbol{A}_{h}, \psi_{h}\right)$ & DOFs for $\left(\boldsymbol{H}_{h}, r_{h}\right)$ & DOFs for $\left(\boldsymbol{u}_{h}, p_{h}\right)$ \\
\hline $\mathcal{T}_{1}$ & 1.732 & 65 & 65 & 60 \\
\hline $\mathcal{T}_{2}$ & 0.866 & 321 & 321 & 408 \\
\hline $\mathcal{T}_{3}$ & 0.433 & 1,937 & 1,937 & 2,976 \\
\hline $\mathcal{T}_{4}$ & 0.217 & 13,281 & 13,281 & 22,656 \\
\hline $\mathcal{T}_{5}$ & 0.108 & 97,985 & 97,985 & 176,640 \\
\hline
\end{tabular}

Example 5.1. This example is to investigate convergence orders of finite element solutions. The physical parameters are set by $R_{e}=R_{m}=\kappa=1$. The tolerances are set by $\delta=10^{-5}$ and $\varepsilon=10^{-6}$. The right-hand sides and the Dirichlet boundary conditions are chosen so that the true solutions are given by

$$
\begin{aligned}
& \boldsymbol{A}=(\sin z, 0,0)^{\top}, \quad \boldsymbol{H}=(0, \cos z, 0)^{\top}, \quad \psi=r=0, \\
& \boldsymbol{u}=(\cos z, \sin (x+z), 0)^{\top}, \quad p=x+y-1 .
\end{aligned}
$$


From Table 2, 3, we find that optimal convergence orders are obtained for physical quantities, namely, $\boldsymbol{A}_{h}, \boldsymbol{H}_{h}, \boldsymbol{u}_{h}$, and $p_{h}$, under their energy norms

$$
\begin{array}{ll}
\left\|\boldsymbol{A}-\boldsymbol{A}_{h}\right\|_{\boldsymbol{H}(\operatorname{curl}, \Omega)} \sim O(h), & \left\|\boldsymbol{H}-\boldsymbol{H}_{h}\right\|_{\boldsymbol{H}(\operatorname{curl}, \Omega)} \sim O(h), \\
\left\|\boldsymbol{u}-\boldsymbol{u}_{h}\right\|_{1, h} \sim O(h), & \left\|p-p_{h}\right\|_{L^{2}(\Omega)} \sim O(h) .
\end{array}
$$

Moreover, we also find that $\left\|\operatorname{div} \boldsymbol{u}_{h}\right\|_{L^{2}(\Omega)}$ is negligible, compared with approximation errors. The reason for $\operatorname{div} \boldsymbol{u}_{h} \neq 0$ is due to the error from solving the system of linear algebraic equations (26), namely, the tolerance $\varepsilon=10^{-6}$.

Table 2: Convergence orders of $\boldsymbol{u}_{h}$ and $p_{h}$. (Example 5.1)

\begin{tabular}{|c|c|c|c|c|c|}
\hline Mesh & $\left\|\boldsymbol{u}-\boldsymbol{u}_{h}\right\|_{1, h}$ & Order & $\left\|p-p_{h}\right\|_{L^{2}(\Omega)}$ & Order & $\left\|\operatorname{div} \boldsymbol{u}_{h}\right\|_{L^{2}(\Omega)}$ \\
\hline $\mathcal{T}_{1}$ & $6.376 \mathrm{e}-01$ & - & $1.227 \mathrm{e}+01$ & - & $3.701 \mathrm{e}-10$ \\
\hline $\mathcal{T}_{2}$ & $2.411 \mathrm{e}-01$ & 1.403 & $4.040 \mathrm{e}-01$ & 4.925 & $2.149 \mathrm{e}-09$ \\
\hline $\mathcal{T}_{3}$ & $1.203 \mathrm{e}-01$ & 1.003 & $1.399 \mathrm{e}-01$ & 1.530 & $5.067 \mathrm{e}-09$ \\
\hline $\mathcal{T}_{4}$ & $5.865 \mathrm{e}-02$ & 1.036 & $5.221 \mathrm{e}-02$ & 1.422 & $2.123 \mathrm{e}-08$ \\
\hline $\mathcal{T}_{5}$ & $2.874 \mathrm{e}-02$ & 1.029 & $2.215 \mathrm{e}-02$ & 1.237 & $6.088 \mathrm{e}-08$ \\
\hline
\end{tabular}

Table 3: Convergence orders of $\boldsymbol{A}_{h}$ and $\boldsymbol{H}_{h}$. (Example 5.1)

\begin{tabular}{|c|c|c|c|c|}
\hline Mesh & $\left\|\boldsymbol{A}-\boldsymbol{A}_{h}\right\|_{\boldsymbol{H}(\mathbf{c u r l}, \Omega)}$ & Order & $\left\|\boldsymbol{H}-\boldsymbol{H}_{h}\right\|_{\boldsymbol{H}(\mathbf{c u r l}, \Omega)}$ & Order \\
\hline $\mathcal{T}_{1}$ & $1.190 \mathrm{e}-01$ & - & $2.114 \mathrm{e}-01$ & - \\
\hline $\mathcal{T}_{2}$ & $5.937 \mathrm{e}-02$ & 1.003 & $9.883 \mathrm{e}-02$ & 1.097 \\
\hline $\mathcal{T}_{3}$ & $2.942 \mathrm{e}-02$ & 1.013 & $4.862 \mathrm{e}-02$ & 1.023 \\
\hline $\mathcal{T}_{4}$ & $1.457 \mathrm{e}-02$ & 1.014 & $2.410 \mathrm{e}-02$ & 1.013 \\
\hline $\mathcal{T}_{5}$ & $7.234 \mathrm{e}-03$ & 1.010 & $1.200 \mathrm{e}-02$ & 1.001 \\
\hline
\end{tabular}

Example 5.2 (Driven cavity flow). The purpose of this example is to demonstrates the optimality of the preconditioned GMRES method for solving a benchmark problem. The external force in the momentum equation is set by $\boldsymbol{f}=\mathbf{0}$. The boundary conditions are set by

$$
\boldsymbol{A}=(0,0,-y), \quad \boldsymbol{H}=(-1,0,0), \quad \boldsymbol{u}=(v, 0,0) \quad \text { on } \partial \Omega,
$$

where $v \in C[0,1]$ and satisfies

$$
v(x, y, 1)=1, \quad v(x, y, z)=0 \quad \forall z \in[0,1-h] .
$$

We fix $\kappa=1, R_{m}=10$ and demonstrate the optimality of the solver for $R_{e}=1,10,100$. The tolerances are set by $\delta=10^{-4}$ and $\varepsilon=10^{-5}$. Let $N_{\text {picard }}$ denote the number of Picard iterations and let $N_{\text {gmres }}$ denote the average number of preconditioned GMRES iterations for solving (26). From Table 4 , we find

\begin{tabular}{|c|c|c|c|}
\hline & \multicolumn{3}{|c|}{$N_{\text {gmres }}\left(N_{\text {picard }}\right)$} \\
\hline${ }_{\text {Mesh }} R_{e}$ & 1 & 10 & 100 \\
\hline $\mathcal{T}_{1}$ & $9(5)$ & $9(4)$ & $7(5)$ \\
\hline $\mathcal{T}_{2}$ & $12(5)$ & $8(4)$ & $8(8)$ \\
\hline $\mathcal{T}_{3}$ & $10(5)$ & $8(5)$ & $10(7)$ \\
\hline $\mathcal{T}_{4}$ & $11(6)$ & $8(5)$ & $14(7)$ \\
\hline $\mathcal{T}_{5}$ & $9(6)$ & $9(6)$ & $15(7)$ \\
\hline
\end{tabular}
that the number of GMRES iterations is quasi-uniform to the meshes for each fixed $R_{e}$. Moreover, the preconditioned GMRES method is robust to $R_{e}$.

Table 4: Robustness and quasi-optimality of the preconditioned GMRES solver. (Example 5.2 ) 
Example 5.3 (Robustness). This example investigates the robustness of the solver to $R_{e}$ and $R_{m}$ by the driven cavity flow in Example 5.2.

We choose $\mathcal{T}_{5}$ as the computational mesh and set $\kappa=\alpha=1$. The tolerances are $\delta=10^{-4}$ and $\varepsilon=10^{-5}$. From Table 5 , we find that, for small $R_{m}$, the solver for linear system is robust with respect to $R_{e}$, while for large $R_{m}$, the number of GMRES iterations grows slightly. Another observation is that with large $R_{m}$, Picard's method is inefficient for solving the nonlinear problem (18). Unfortunately, the present discretization using upwinding in the convection term $\boldsymbol{w} \cdot \nabla \boldsymbol{u}$, which makes the Newton's method difficult to use. In the future, acceleration techniques in optimization field for nonlinear iteration can be incorporated to improve the nonlinear convergence rate. In Figure 1, we depict the streamlines of $\boldsymbol{u}_{h}$ projected onto the cross-section at $y=0.5$ for different values of $R_{m}$.

Table 5: Sensitivity to $R_{e}$ and $R_{m}$. (Example 5.3)

\begin{tabular}{|c|c|c|c|c|}
\hline & \multicolumn{4}{|c|}{$N_{\text {gmres }}\left(N_{\text {picard }}\right)$} \\
\hline$R_{e}$ & 1 & 20 & 40 & 60 \\
\hline 1 & $6(4)$ & $10(11)$ & $12(26)$ & $14(>100)$ \\
\hline 10 & $5(5)$ & $11(10)$ & $13(30)$ & $17(>100)$ \\
\hline 100 & $8(10)$ & $20(8)$ & $31(44)$ & $39(>100)$ \\
\hline
\end{tabular}
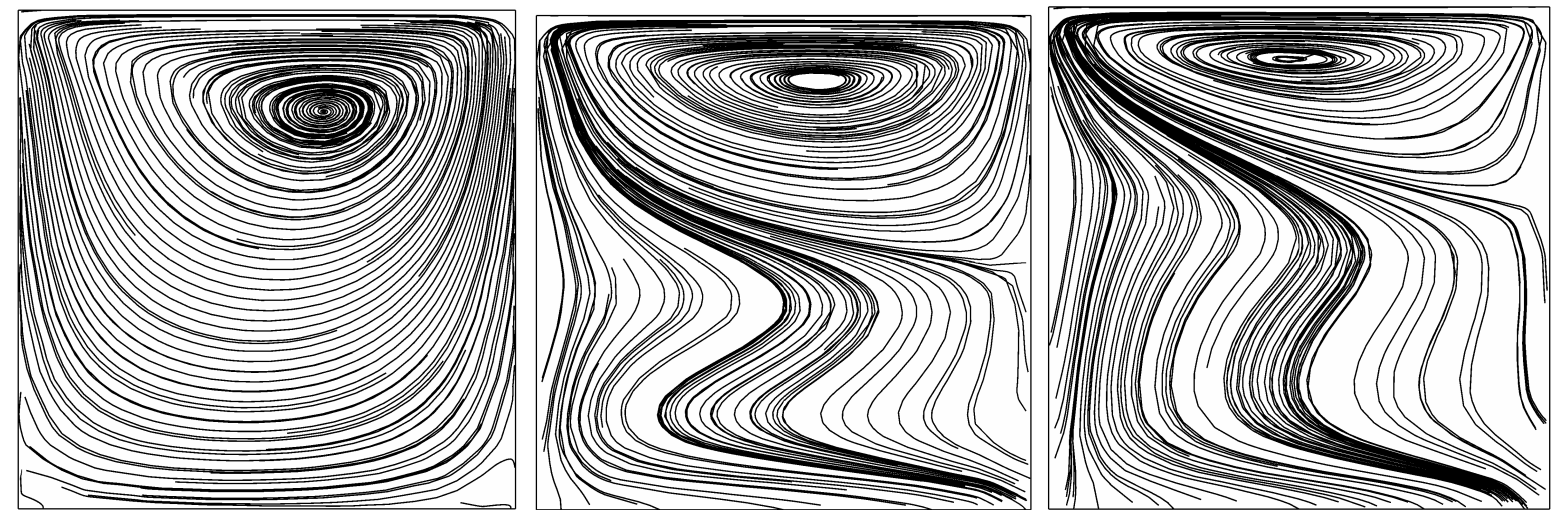

Figure 1: Projections of the streamlines of $\boldsymbol{u}_{h}$ on the cross section $y=0.5$ with $R_{e}=100$ and $\kappa=1$ (from left to the right $\left.R_{m}=1,10,50\right)$. (Example 5.3)

Next we choose $\mathcal{T}_{5}$ as the computational mesh and set $\alpha=1, R_{e}=100$. We examine the effect of the magneto-fluid coupling term on the performance of the preconditioner. Remember from (36) that the approximate matrix of $\tilde{\mathbb{F}}$ is $\mathbb{S}_{u} \approx \mathbb{F}+\mathbb{J H}^{-1} \mathbb{J}^{\top}$. The data in Table 6 is the result with the proposed preconditioner, while in Table 7 we ignore the coupling term $\mathbb{J H}^{-1} \mathbb{J}^{\top}$ and only use $\mathbb{F}$ as the approximate matrix. From Table 7, we see that the numbers of the GMRES increase considerably for large $\kappa$ or $R_{m}$.

Table 6: Performance for different $\kappa$ and $R_{m}$ with approximation $\mathbb{S}_{u}$. (Example 5.3 )

\begin{tabular}{|c|c|c|r|}
\hline & \multicolumn{3}{|c|}{$N_{\text {gmres }}\left(N_{\text {picard }}\right)$} \\
\hline \multirow{2yyy}{*}{$R_{m}$} & 1 & 20 & \multicolumn{1}{|c|}{40} \\
\hline 1 & $8(10)$ & $20(8)$ & $31(44)$ \\
\hline 20 & $24(7)$ & $70(6)$ & $90(7)$ \\
\hline 40 & $32(7)$ & $87(6)$ & $109(6)$ \\
\hline 60 & $38(7)$ & $99(6)$ & $125(6)$ \\
\hline
\end{tabular}

Example 5.4. This example investigates the sensitivity of the preconditioner to the grad-div stabilization parameter $\alpha$ by the driven cavity flow in Example 5.2. 
Table 7: Performance for different $\kappa$ and $R_{m}$ with approximation $\mathbb{F}$. (Example 5.3)

\begin{tabular}{|c|c|c|c|}
\hline & \multicolumn{3}{|c|}{$N_{\text {gmres }}\left(N_{\text {picard }}\right)$} \\
\hline$R_{m}$ & 1 & 20 & 40 \\
\hline 1 & $7(10)$ & $26(7)$ & $37(47)$ \\
\hline 20 & $24(7)$ & $119(6)$ & $171(8)$ \\
\hline 40 & $35(7)$ & $161(6)$ & $171(7)$ \\
\hline 60 & $43(7)$ & $167(6)$ & $175(8)$ \\
\hline
\end{tabular}

We fix $R_{m}=1$ and $\kappa=100$ and investigate the performance of the GMRES solver to $R_{e}$ and $\alpha$. From Table 8 , we find that,

- for $\alpha \geq 0.5$, the convergence of the solver is not sensitive to $\alpha$,

- while for $\alpha=0$, the number of GMRES iterations increases fast with $R_{e}$.

We conclude that the grad-div stabilization plays an important role in the performance of the block preconditioner.

Table 8: Sensitivity to $R_{e}$ and $\alpha$. (Example 5.4)

\begin{tabular}{|c|c|c|c|c|c|}
\hline & \multicolumn{5}{|c|}{$N_{\text {gmres }}\left(N_{\text {picard }}\right)$} \\
\hline$R_{e}$ & 0 & 0.5 & 1 & 10 & 100 \\
\hline 1 & $13(4)$ & $11(4)$ & $10(4)$ & $9(4)$ & $7(5)$ \\
\hline 10 & $29(5)$ & $25(5)$ & $21(5)$ & $15(4)$ & $12(4)$ \\
\hline 100 & $105(6)$ & $46(6)$ & $43(6)$ & $34(6)$ & $29(6)$ \\
\hline
\end{tabular}

\section{Conclusions}

In this paper, we propose a monolithic constrained transport finite element method for stationary incompressible MHD equations. The discrete velocity, discrete current density, and discrete magnetic induction are all divergence-free in the momentum equation, especially in the Lorentz force. Based on an augmented Lagrangian block preconditioner, we also develop a preconditioned GMRES solver for the linearized system of algebraic equations in every Picard iteration. Although the present work only presents first-order discretization for $\boldsymbol{u}, \boldsymbol{H}$, and $\boldsymbol{A}$, the method can be extended to high-order finite elements straightforwardly. The monolithic manner can be applied to time-dependent MHD equations to develop fully implicit method, which will permit large time-step length and stable long time simulation compared with explicit method.

\section{Acknowledgments}

The computations were (partly) done on the high performance computers of State Key Laboratory of Scientific and Engineering Computing, Chinese Academy of Science.

Lingxiao Li was supported by National Natural Science Foundation of China under Grant 11901042. Weiying Zheng was supported in part by the National Science Fund for Distinguished Young Scholars 11725106 and by China NSF grant 11831016.

\section{References}

[1] C. Amrouche, C. Bernardi, M. Dauge and V. Girault. Vector potentials in three-dimensional nonsmooth domains. Math. Methods Appl. Sci., 21(1998), 9, pp. 823-864.

[2] J. H. Adler, T. R. Benson, E. C. Cyr, S. P. MacLachlan and R. Tuminaro. Monolithic Multigrid Methods for Two-Dimensional Resistive Magnetohydrodynamics. SIAM J. Sci. Comput., 38(1), 2016, pp. B1-B24. 
[3] M. Benzi and M. A. Olshanskii. An Augmented Lagrangian-Based Approach to the Oseen Problem. SIAM J. Sci. Comput., 28(6), 2006, pp. 2095-2113.

[4] M. Benzi and M. A. Olshanskii. Field-of-values convergence analysis of augmented Lagrangian preconditioners for the linearized Navier-Stokes problem. SIAM J. Numer. Anal., 49, 2011, pp. 770-788.

[5] M. Benzi and Z. Wang. Analysis of Augmented Lagrangian-Based Preconditioners for the Steady Incompressible Navier-Stokes Equations. SIAM J. Sci. Comput., 33(5), 2011, pp. 2761-2784.

[6] J. U. Brackbill and D.C. Barnes. The effect of nonzero $\nabla \cdot B$ on the numerical solution of the magnetohydrodynamic equations. J. Comput. Phys., 35, 1980, pp. 426-430.

[7] D. S. Balsara and M. Dumbser. Divergence-free MHD on unstructured meshes using high order finite volume schemes based on multidimensional Riemann solvers. J. Comput. Phys., 299, 2015, pp. $687-715$

[8] S. Badia, A. F. Martín and R. Planas. Block recursive LU preconditioners for the thermally coupled incompressible inductionless MHD problem. J. Comput. Phys., 274, 2014, pp. 562-591.

[9] L. Chacón. An optimal, parallel, fully implicit Newton-Krylov solver for three-dimensional viscoresistive magnetohydrodynamics. Physics of Plasmas, 15, 056103(2008).

[10] B. Cockburn, G. Kanschat and D. Schötzau. A Note on Discontinuous Galerkin Divergence-free Solutions of the Navier-Stokes equations. J. Sci. Comput., 31(1), 2007, pp. 61-73.

[11] X.-C. Cai and M. Sarkis. A Restricted Additive Schwarz Preconditioner for General Sparse Linear Systems. SIAM J. Sci. Comput., 21(2), 1999, pp. 792-797.

[12] P. A. Davidson. An Introduction to Magnetohydrodynamics. Cambridge Texts in Applied Mathematics. 2001.

[13] H. Elman, D. Silvester and A. Wathen. Finite Elements and Fast Iterative Solvers: With Applications in Incompressible Fluid Dynamics(Second Edition). Oxford University Press, 2014.

[14] C. R. Evans and J. F. Hawley. Simulation of magnetohydrodynamic flows: A Constrained Transport Method. Astrophysical Journal, 332, 1988, pp. 659-677.

[15] P. E. Farrell, L. Mitchell and F. Wechsung. An Augmented Lagrangian preconditioner for the 3D stationary incompressible Navier-Stokes Equations at High Reynolds number. SIAM J. Sci. Comput., Vol. 41, No. 5, 2019, pp. A3073-A3096.

[16] K. Felker and J. M. Stone. A fourth-order accurate finite volume method for ideal MHD via upwind constrained transport. J. Comput. Phys., 375, 2018, pp. 1365-1400.

[17] J.-F. Gerbeau, C. Le Bris and T. Lelièvre. Mathematical Methods for the Magnetohydrodynamics of Liquid Metals. New York: Oxford University Press, 2006.

[18] J.-F. Gerbeau. A stabilized finite element method for the incompressible magnetohydrodynamic equations. Numer. Math., 87, 2000, pp. 83-111.

[19] V. Girault and P.-A. Raviart. Finite Element Methods for Navier-Stokes Equations, Theory and Algorithms. Springer-Verlag, 1986.

[20] C. Greif, D. Li, D. Schötzau and X. Wei. A mixed finite element method with exactly divergence-free velocities for incompressible magnetohydordynamics. Computer Methods in Applied Mechanics and Engineering, 199(45), 2010, pp. 2840-2855.

[21] C. Greif and D. Schötzau. Preconditioners for the discretized time-harmonic Maxwell equations in mixed form. Numer. Linear Algebra Appl., 14, 2007, pp. 281-297.

[22] M. D. Gunzburger, A.J. Meir and J. S. Peterson. On the existence, uniqueness, and finite element approximation of solutions of the equations of stationary, incompressible magnetohydrodynamics. Math. Comp., 56, 1991, pp. 523-563.

[23] J. Hawley and J. Stone. MOCCT: A numerical technique for astrophysical MHD. Computer Physics Communications, Vol 89, 1995, pp. 127-148. 
[24] R. Hiptmair, L. Li, S. Mao and W. Zheng. A fully divergence-free Finite Element method for Magnetohydrodynamic equations. Mathematical Models and Methods in Applied Sciences, 28(4), 2018, pp. 659-695.

[25] R. Hiptmair. Finite elements in computational electromagnetism. Acta Numerica, Vol. 11, 2002, pp. 237-339.

[26] R. Hiptmair and J. Xu. Nodal Auxiliary Space Preconditioning in $H($ curl) and $H($ div) Spaces. SIAM J. Numer. Anal., 45(6), 2007, pp. 2483-2509.

[27] Y. He. Unconditional convergence of the Euler semi-implicit scheme for the three-dimensional incompressible MHD equations. IMA J. Numer. Anal., 35, 2015, pp. 767-801.

[28] V. E. Henson and U. M. Yang. BoomerAMG: a parallel algebraic multigrid solver and preconditioner. Applied Numerical Mathematics, Vol. 41, 2002, pp. 155-177.

[29] K. Hu, Y. Ma and J. Xu. Stable finite element methods preserving $\nabla \cdot B=0$ exactly for MHD models. Numer. Math., 135, 2017, pp. 371-396.

[30] V. John, A. Linke, C. Medron, M. Neilan and L. G. Rebholz. On the divergence constraint in Mixed Finite Element Methods for Incompressible Flows. SIAM Review, 2017, Vol. 59, pp. 492-544.

[31] S. Jardin. Computational Methods in Plasma Physics. CRC Press, 2010.

[32] L. Li and W. Zheng. A robust solver for the finite element approximation of stationary incompressible MHD equations in 3D. J. Comput. Phys., 351(2017), pp. 254-270.

[33] L. Li, M.-J. Ni and W. Zheng. A charge-conservative Finite Element Method for inductionless MHD equations. Part I: Convergence. SIAM J. Sci. Comput., 41(4), 2019, pp. B796-B815.

[34] P. T. Lin, J. N. Shadid, J. J. Hu, R. P. Pawlowski and E. C. Cyr. Performance of fully-coupled algebraic multigrid preconditioners for large-scale VMS resistive MHD. Journal of Computational and Applied Mathematics, Vol. 344, 2018, pp. 782-793.

[35] P. Monk. Finite Element Methods for Maxwell's Equations. Oxford University Press, 2003.

[36] J. C. Nédélec. A new family of mixed finite elements in $\mathbb{R}^{3}$. Numer. Math., 50(1), 1986, pp. 57-81.

[37] M.-J. Ni, R. Munipalli, P. Huang, N. B. Morley and M. A. Abdou. A current density conservative scheme for incompressible MHD flows at low magnetic Reynolds number. Part II: On an arbitrary collocated mesh. J. Comput. Phys., 227(2007), pp. 205-228.

[38] M.-J. Ni and J.-F. Li. A consistent and conservative scheme for incompressible MHD flows at a low magnetic Reynolds number. Part III: On a staggered mesh. J. Comput. Phys., 231, 2012, pp. 281-298.

[39] M. A. Olshanskii and A. Reusken. Grad-div stabilization for Stokes equations. Math. Comp., 73, 2004, pp. 1699-1718.

[40] E. Phillips, H. Elman, E. Cyr, J. Shadid and R. Pawlowski. A Block Preconditioner for an exact penalty formulation for stationary MHD. SIAM J. Sci. Comput., Vol. 36, No. 6, 2014, pp. B930-B951.

[41] E. Phillips, J. Shadid, E. Cyr, H. Elman and R. Pawlowski. Block Preconditioners for stable mixed nodal and edge finite element representations of incompressible Resistive MHD. SIAM J. Sci. Comput., Vol. 38, 2016, pp. B1009-B1031.

[42] B. Philip, L. Chacón and M. Pernice. Implicit Adaptive Mesh Refinement for 2D Reduced Resisitive Magnetohydrodynamics. J. Comput. Phys., Vol. 227, 2008, pp. 8855-8874.

[43] A. Prohl. Convergent finite element discretizations of the nonstationary incompressible magnetohydrodynamics system. ESAIM: M2AN., 42(6), 2008, pp. 1065-1087.

[44] J. A. Rossmanith. An Unstaggered, High-Resolution constrained transport method for magnetohydrodynamic flows. SIAM J. Sci. Comput., Vol.28, 2006, pp. 1766-1797. 
[45] J. D. Ramshaw. A Method for Enforcing the Solenoidal Condition on Magnetic Field in Numerical Calculation. J. Comput. Phys., 52, 1983, pp. 592-596.

[46] W. Ruan, C. Xia and R. Keppens. Extreme-ultraviolet and X-Ray Emission of Turbulent Solar Flare Loops. Astrophysical Journal Letters, 2019, 877, L11.

[47] N. B. Salah, A. Soulaimani and W. G. Habashi. A finite element method for magnetohydrodynamics. Computer Methods in Applied Mechanics and Engineering, 190(43), 2001, pp. 5867-5892.

[48] J. N. Shadid, R. P. Pawlowski, J. W. Banks, L. Chacón, P. T. Lin and R. Tuminaro. Towards a scalable fully-implicit fully-coupled resistive MHD formulation with stabilized FE methods. J. Comput. Phys., 229, 2010, pp. 7649-7671.

[49] H. Su, S. Mao and X. Feng. Optimal Error Estimates of Penalty Based Iterative Methods for Steady Incompressible Magnetohydrodynamics Equations with Different Viscosities. J. Sci. Comput., Vol. 79, 2019, pp. 1078-1110.

[50] J. N. Shadid, R. P. Pawlowski, E. C. Cyr, R. S. Tuminaro, L. Chacón and P. D. Weber. Scalable implicit incompressible resistive MHD with stabilized FE and fully-coupled Newton-Krylov-AMG. Comput. Methods Appl. Mech. Engre., Vol. 304, 2016, pp. 1-25.

[51] A. Schneebeli and D. Schötzau. Mixed finite elements for incompressible magneto-hydrodynamics. Comptes Rendus Mathematique, Vol. 337, 2003, pp. 71-74.

[52] D. Schötzau. Mixed finite element methods for stationary incompressible magneto-hydrodynamics. Numer. Math., 96, 2004, pp. 771-800.

[53] G. Tóth. The $\nabla \cdot B=0$ Constraint in Shock-Capturing Magnetohydrodynamics Codes. J. Comput. Phys., Vol. 161, 2000. pp. 605-652.

[54] J. Zhang and M.-J. Ni. A consistent and conservative scheme for MHD flows with complex boundaries on an unstructured Cartesian adaptive system. J. Comput. Phys., 256, 2014, pp. 520-542.

[55] L. Zhang. A Parallel Algorithm for Adaptive Local Refinement of Tetrahedral Meshes Using Bisection. Numer. Math.: Theory, Methods and Applications, 2, 2009, pp. 65-89. 